$\mathrm{R} / 95 / 13$

hep-th/9505032

May, 1995, Revised December 1995

\title{
NEW SUPERGRAVITIES WITH CENTRAL CHARGES AND KILLING SPINORS IN 2+1 DIMENSIONS
}

\author{
P.S. HowE \\ Department of Mathematics, King's College London, \\ London, U.K. \\ and \\ J.M. IzQuierdo, G. PAPAdopoulos and P. K. Townsend \\ DAMTP, University of Cambridge, \\ Silver Street, Cambridge CB3 9EW, U.K.
}

\begin{abstract}
We construct a new class of $(p, q)$-extended Poincaré supergravity theories in $2+1$ dimensions as Chern-Simons theories of supersymmetry algebras with both central and automorphism charges. The new theories have the advantage that they are limits of corresponding $(p, q)$ adS supergravity theories and, for not too large a value of $N=p+q$, that they have a natural formulation in terms of offshell superfields, in which context the distinction between theories having the same value of $N$ but different $(p, q)$ arises because of inequivalent conformal compensator superfields. We also show that, unlike previously constructed N-extended Poincaré supergravity theories, the new $(2,0)$ theory admits conical spacetimes with Killing spinors. Many of our results on $(2,0)$ Poincaré supergravity continue to apply in the presence of coupling to $\mathrm{N}=2$ supersymmetric sigma-model matter.
\end{abstract}




\section{Introduction}

It is now widely appreciated that (super)gravity theories in $2+1$ dimensions can be interpreted as Chern-Simons (CS) theories of the appropriate (super)algebra $[1,2,3]$. For example, the N-extended Poincaré supergravity of Marcus and Schwarz [4] can be interpreted [5] as the CS theory of the standard N-extended superalgebra spanned by the spinor supercharges $Q^{i}, i=1 \ldots N$, the 3 -momentum $P_{a}$ and angular 3-momentum $M_{a}$. For $\mathrm{N}=1$ this algebra is the only one permitted by the Haag-Lopuszanski-Sohnius theorem, but for $N \geq 2$ there is the possibility of including central charges and it is natural to wonder whether this could lead to new Poincaré supergravity theories. Consider the $N=2$ case: in addition to the usual super-Poincaré generators one can introduce a central charge $Z$ such that

$$
\left\{Q_{\alpha}^{i}, Q_{\beta}^{j}\right\}=-\frac{1}{2} \delta^{i j}\left(\gamma^{a}\right)_{\alpha \beta} P_{a}+i \epsilon_{\alpha \beta} \epsilon^{i j} Z
$$

A problem with this algebra in the context of a CS formulation of $\mathrm{N}=2$ Poincaré supergravity is that it does not admit an invariant non-degenerate inner product. This difficulty can be overcome as follows. We first observe that this superalgebra has an $S O(2)$ group of automorphisms. Let $T$ be the $S O(2)$ generator; then the only non-trivial commutator with $T$ is

$$
\left[T, Q_{\alpha}^{i}\right]=-\epsilon^{i j} Q_{\alpha}^{j}
$$

We then observe that the extension ${ }^{\star}$ of the $s o(2)$ algebra by the $\mathrm{N}=2$ Poincaré superalgebra with central charge does have an invariant non-degenerate inner product; in fact, it has a three-parameter family of such inner products. Let $C$ and $A$ be the one-form gauge potentials associated to $Z$ and $T$ respectively. Then, for any choice of the inner product, the corresponding CS $N=2$ supergravity theory

\footnotetext{
^ We use the word 'extension' in its technical sense.
} 
includes the new term

$$
\int C d A
$$

in addition to the usual Einstein-Hilbert and Rarita-Schwinger terms. For reasons which will be explained shortly, we shall refer to the Marcus-Schwarz (MS) $N=2$ theory as the $(1,1)$ Poincaré supergravity and the new $N=2$ theories as $(2,0)$ Poincaré supergravity theories. This whole discussion can be generalized to $N>2$ and leads to the subdivision of the $N=p+q$ Poincaré supergravity theory into the $(\mathrm{p}, \mathrm{q})$-Poincaré supergravity theories. We present this more general construction in section 7. One motivation for the introduction of central charges in the Poincaré superalgebra comes from consideration of the (p,q) anti de Sitter (adS) supergravity theories, which include an $S O(p) \times S O(q)$ CS term and a cosmological constant proportional to $m^{2}$, where $m$ is a gravitino 'mass' parameter. The $(\mathrm{p}, \mathrm{q})$-extended Poincaré superalgebra is found in the limit as $m \rightarrow 0$. Even so, the $m \rightarrow 0$ limit of the (p,q) adS supergravity action of [2] is problematic when either $p$ or $q$ is greater than one; the only non-singular way to take the $m \rightarrow 0$ limit causes the $S O(p) \times S O(q)$ gauge fields to disappear from the action (which is then the $\mathrm{N}=\mathrm{p}+\mathrm{q}$ extended MS Poincaré supergravity) but leaves a non-zero supersymmetry transformation for them [5]. This means that the incorporation of a cosmological constant into the $N>2$ MS theories cannot be done without the introduction of additional fields, which is a state of affairs that could never arise from elimination of auxiliary fields in an off-shell supergravity theory. One purpose of this paper is to show how this difficulty is resolved by consideration of a trivial extension of the $(\mathrm{p}, \mathrm{q})$ adS superalgebra by an $s o(p) \oplus s o(q)$ algebra. A particular $m \rightarrow 0$ limit of these new (p,q) adS supergravity theories yields the (p,q) Poincaré ones. Thus the $(\mathrm{p}, \mathrm{q})$ Poincaré supergravity theories introduced here naturally arise as limits of $(p, q)$ adS supergravity theories. Another motivation for the new CS formulation of Poincaré and adS supergravities comes from consideration of off-shell supersymmetry. Since it is obvious that the algebra of CS gauge transformations already closes off-shell, i.e. without the use of the field equations, and that these transformations 
include spinorial ones, it might be thought that there is nothing to be considered here. However, it is easy to verify that the numbers of off-shell boson and fermion fields (subtracting the gauge degrees of freedom) are not generally equal, which is puzzling since this is usually thought to be a precondition of off-shell supersymmetry. The resolution of this puzzle lies in the correspondence between the gauge symmetries of the CS theory and the geometrical symmetries of the supergravity theory, which contain spacetime diffeomorphisms. This correspondence is an on-shell one [5], so that off-shell closure of the CS gauge transformations does not imply off-shell closure of the geometrical supersymmetry transformations. It is the latter that is needed for a superspace formulation, and this requires equality of off-shell boson and fermion degrees of freedom. This shows, incidentally, that there are, in principle, two ways in which off-shell closure of the algebra of local supersymmetry transformations may be achieved in theories for which given supersymmetry transformations form a closed algebra only on-shell. One can try to close the algebra by the addition of 'trivial' transformations or one can try to close it by the addition of auxiliary fields, which is tantamount to a reformulation in terms of superfields. The former method was actually advocated in an early attempt to obtain a closed off-shell algebra for $N=1$ supergravity in $3+1$ dimensions [6] but the attempt was unsuccessful because in spacetimes of dimension $3+1$, or higher, the addition of auxiliary fields is unavoidable. In $2+1$ dimensions one can always close the algebra in pure supergravity theories by adding 'trivial' gauge transformations and this amounts to a reformulation as a CS theory, but this is unlikely to work once matter is included. We shall consider the supersymmetry algebra to be closed off-shell only if the geometrical supersymmetry transformations form a closed algebra, which effectively means that we consider a given theory to be offshell supersymmetric only if it has an off-shell superfield formulation. It is by no means guaranteed that such a formulation exists; considerable difficulties appear for $(\mathrm{p}, \mathrm{q})$ theories when either $p$ or $q$ exceeds 2 , as we shall see. To explain why off-shell supersymmetry is relevant to $(p, q)$ Poincaré supergravity it is simplest to consider the $\mathrm{N}=2$ case, which subdivides into the $(2,0)$ and the $(1,1)$ cases. The 
possibility of two inequivalent theories arises in the $\mathrm{N}=2$ superfield context from the fact that the conformal invariance of the conformal supergravity supermultiplet can be 'compensated', to produce a Poincaré supermultiplet, by two inequivalent 'compensating superfields'. The $\mathrm{N}=2$ conformal supergravity supermultiplet has the field [dimension] content [7]:

$$
e_{m}{ }^{a}[0] ; \psi_{m}^{i}\left[\frac{1}{2}\right] ; A_{m}[1]
$$

where $e_{m}{ }^{a}$ is the dreibein, $\psi_{m}^{i}$ the two gravitini, $A_{m}$ the $\mathrm{SO}(2)$ gauge potential and the dimension is the 'geometrical' one. One possible $\mathrm{N}=2$ compensator multiplet has the field [dimension] content

$$
A[0], B[0] ; \lambda^{i}\left[\frac{1}{2}\right] ; \tilde{K}_{i j}[1],
$$

where $\tilde{K}_{i j}$ is symmetric and traceless in its two $\mathrm{SO}(2)$ indices. The scalars $A$ and $B$ become the trace of the dreibein and the longitudinal component of $A_{m}$, respectively, while $\lambda^{i}$ becomes the gamma-trace of $\psi_{m}^{i}$. This yields the Poincaré supermultiplet with the following field [dimension] content

$$
e_{m}{ }^{a}[0] ; \psi_{m}^{i}\left[\frac{1}{2}\right] ; A_{m}[1], \tilde{K}_{i j}[1]
$$

where $A_{m}$ and $\tilde{K}_{i j}$ are both auxiliary fields. The off-shell Poincaré and adS supergravity theories constructed from this supermultiplet are those of $(1,1)$ supersymmetry [8]. Another possible compensating supermultiplet has the field [dimension] content

$$
A[0], C_{m}[0] ; \lambda^{i}\left[\frac{1}{2}\right] ; K[1] .
$$

The scalar $A$ and spinors $\lambda^{i}$ get absorbed as before, so that the field [dimension] content of the resulting Poincaré supermultiplet is

$$
e_{m}{ }^{a}[0], C_{m}[0] ; \psi_{m}^{i}\left[\frac{1}{2}\right] ; A_{m}[1], K[1],
$$

where the scalar $K$ is auxiliary but the vectors $A_{m}$ and $C_{m}$ are both gauge fields which can be identified as the 'Automorphism' and 'Central' gauge fields of the 
$(2,0)$ Poincaré supergravity, i.e. the components of the gauge potential one forms $A$ and $C$ introduced above. Indeed, one finds that the off-shell supersymmetric action contains precisely the term (1.3), as expected for the $(2,0)$ theory. There is a precedent for such a term in the new-minimal formulation of off-shell $\mathrm{N}=1 \mathrm{su}-$ pergravity in $3+1$ dimensions, where $C_{m}$ is replaced by a two-form gauge potential $[9,10,11]$. In that case, it is customary to consider the $C$ and $A$ gauge fields as auxiliary, despite the fact that their field equations are not algebraic, because on-shell they are pure gauge. While this is justifiable in $3+1$ dimensions it is obviously not in $2+1$ dimensions because, by the same criterion, all CS gauge fields, including the dreibein, would have to be considered as auxiliary. We may conclude from this that in $2+1$ dimensions different choices of the conformal compensator supermultiplets can lead to inequivalent Poincaré theories. As for all supergravity theories, the gravitino supersymmetry transformation of an $\mathrm{N}=2$ Poincaré theory is a covariant derivative of the supersymmetry parameter $\zeta$. In complex spinor notation we can write it as $\delta_{\zeta} \psi=\mathcal{D} \zeta$ where $\mathcal{D}$ is a covariant derivative acting on complex spinors. For the $(1,1)$ Poincaré theory, and in a purely bosonic background, $\mathcal{D}$ is just the standard covariant derivative constructed from the spin connection. One can define a supersymmetric spacetime to be one for which all fermions and their supersymmetry variations vanish for some non-zero supersymmetry parameter. A necessary condition is therefore that there exist a nonzero spinor $\zeta$ such that $\mathcal{D} \zeta=0$. Since this equation is linear in $\zeta$, its consequences are unchanged if we replace the anticommuting spinor $\zeta$ by a commuting one $\kappa$. Such a spinor, satisfying $\mathcal{D} \kappa=0$, is generally called a Killing spinor. In the context of $(1,1)$ supergravity a Killing spinor is one that is covariantly constant with respect to the usual spin-connection, which is possible only if the spacetime is flat. Solutions of the vacuum field equations of all Poincaré supergravity theories outside a matter source are pure gauge configurations on conical spacetimes with a deficit angle equal to the mass $M$ in the interior; we therefore restrict $M$ to lie in the range $0 \leqslant M<2 \pi$. Since these spacetimes are flat one might expect them to be supersymmetric but, as recently observed [12], there are no covariantly constant spinors in conical spacetimes unless 
$M=0$, so the only supersymmetric spacetime in the context of the $(1,1)$ Poincaré supergravity is $2+1$ Minkowski spacetime, with $M=0$, and, evidently, the same conclusion holds for the N-extended MS supergravity theories for any $N$. Another purpose of this paper is to point out that this conclusion changes when one considers the new $(2,0)$ Poincaré supergravity. Here the covariant derivative $\mathcal{D}$ includes the automorphism gauge field $A$. The corresponding charge, $Q$, can be chosen, without loss of generality, to satisfy $|Q|<\pi$. It is now possible to find spinors that are covariantly constant outside the region containing the matter provided that

$$
\text { either } \quad M=2|Q| \quad \text { or } \quad M=2|Q \pm \pi| \text {, }
$$

depending on whether the spin structure is even or odd, respectively. In particular, conical point-particle spacetimes admit Killing spinors for any allowed value of $M$, and multi point-particle spacetimes may do so also, provided a condition analogous to (1.9) holds for every particle. Matter coupling to N-extended supergravity theories with zero cosmological constant has been considered in [17] and with non-zero cosmological constant for $\mathrm{N}=2$ in [13]. More recently, an $\mathrm{N}=2$ matter coupled three-dimensional supergravity was constructed by dimensional reduction from four dimensions [18], and the possibility of Killing spinors in this context was demonstrated. Here we consider the coupling of $(2,0)$ Poincaré supergravity to sigma-model matter. An interesting feature of this model is that while the automorphism gauge field $A$ couples to the $U(1)$ Noether current, the central charge gauge field $C$ couples to a topological current. In supergravity theories one generally finds that supersymmetric spacetimes, i.e. those admitting Killing spinors, saturate a bound on the mass in terms of the charges. Of course, supergravity theories in $2+1$ dimensions are exceptional in many respects, but such a bound has recently been established for adS supergravity theories in $2+1$ dimensions [13]. This might lead one to suppose that (1.9) has an interpretation as the saturation by supersymmetric spacetimes of a lower bound on the mass $M$ of any spacetime solving the equations of a matter-coupled $(2,0)$ Poincaré supergravity, for a given choice of spin structure for the gravitino. The standard way of deriving a bound 
on the mass in terms of the charges in supergravity theories proceeds via a generalization [14] of the spinorial proof of the positive energy theorem. A curious feature of the attempt, which we describe later, to obtain such a bound in the present case is that the 'Witten condition' that must be imposed on the spinor appearing in the expression for the total energy [15] turns out to be equivalent to the condition that this spinor be a Killing spinor. Thus, instead of deriving a bound one merely confirms the equality (1.9). The organisation of this paper is as follows: In section 2 we describe the CS formulation of the $(2,0)$ Poincaré supergravity theories. In section 3 we discuss Killing spinors of the $(2,0)$ Poincaré supergravity theory. In section 4 we couple the $(2,0)$ supergravity theory to sigma-model matter. In section 5 we discuss the application of the Witten-Nester method to $2+1$ gravity, and prove an energy 'equality' theorem. In section 6 we explain the connection with off-shell superfields and present the superfield construction of $(2,0)$ Poincaré supergravity coupled to the most general sigma-model matter. In sections 7 and 8 we present the CS formulation of the general (p,q) Poincaré supergravity and discuss their relation to (p,q) adS supergravity.

\section{CS formulation of $(2,0)$ Poincaré Supergravity}

The non-trivial commutators of the $(2,0)$ Poincaré superalgebra and its $s o(2)$ automorphism algebra are

$$
\begin{aligned}
{\left[M_{a}, M_{b}\right] } & =-\epsilon_{a b}^{c} M_{c} \\
{\left[M_{a}, P_{b}\right] } & =-\epsilon_{a b}{ }^{c} P_{c} \\
\left\{Q_{\alpha}^{i}, Q_{\beta}^{j}\right\} & =-\frac{1}{2} \delta^{i j}\left(\gamma^{a}\right)_{\alpha \beta} P_{a}+i \epsilon_{\alpha \beta} \epsilon^{i j} Z \\
{\left[T, Q_{\alpha}^{i}\right] } & =-\epsilon^{i j} Q_{\alpha}^{j} .
\end{aligned}
$$

We use the 'mostly-minus' metric convention and hence gamma matrices that are pure imaginary. Note that

$$
\gamma^{a} \gamma^{b}=\eta^{a b}+i \epsilon^{a b c} \gamma_{c} \quad(\bar{\psi})_{\alpha}=\psi^{\beta} \epsilon_{\beta \alpha}
$$

We also introduce a formal conjugation with respect to which all the even genera- 
tors of the superalgebra are antihermitian whereas the odd ones are hermitian, and we adopt the standard convention that complex conjugation of a fermion bi-linear introduces an additional minus sign. Defining the (antihermitian) connection * one-form $a$ by

$$
a=e^{a} P_{a}+\omega^{a} M_{a}+C Z+A T+\psi^{i} Q_{i}
$$

we compute the curvature two-form $F=d a+a^{2}$ to be

$$
F=T^{a} P_{a}+F^{a}(\omega) M_{a}+F(C) Z+F(A) T+\mathcal{D} \psi^{i} Q_{i}
$$

where

$$
\begin{aligned}
T^{a} & =d e^{a}-\epsilon_{b c}^{a} \omega^{b} e^{c}-\frac{1}{4} \psi^{i} \gamma^{a} \psi^{i} \\
F^{a}(\omega) & =d \omega^{a}-\frac{1}{2} \epsilon_{b c}^{a} \omega^{b} \omega^{c} \\
F(C) & =d C \\
F(A) & =d A \\
\mathcal{D} \psi^{i} & =d \psi^{i}+\frac{i}{2} \omega^{c} \gamma_{c} \psi^{i}+A \epsilon^{i j} \psi^{j} .
\end{aligned}
$$

There is a three-parameter non-degenerate inner product on the above algebra, which is given as a special case of the four-parameter inner product for the general N-extended case in section 5. The freedom represented by the choice of these parameters is not significant for the classical theory considered here, so we shall make the 'simplest' choice for which the only non-zero components of the inner product, in the above basis, are

$$
\left\langle M_{a}, P_{b}\right\rangle=\mu \eta_{a b} \quad\left\langle Q_{\alpha}^{i}, Q_{\beta}^{j}\right\rangle=i \mu \epsilon_{\alpha \beta} \delta^{i j} \quad\langle T, Z\rangle=-\mu
$$

where $\mu$ is a real non-zero constant with dimensions of mass and the inner product is hermitian with respect to the formal conjugation introduced above. The CS

\footnotetext{
$\star$ Note that in our conventions the field $\psi$ anticommutes with $Q$.
} 
action is then

$$
S=\mu \int d^{3} x\left[e R-i \varepsilon^{m n p} \bar{\psi}_{m}^{i} \mathcal{D}_{n} \psi_{p}^{i}+2 \varepsilon^{m n p} C_{m} \partial_{n} A_{p}\right]
$$

where we consider the spin-connection $\omega_{m}{ }^{a}$ to be an independent field, i.e. this is the first-order form of the supergravity action. This action is invariant under the gauge transformations of the connection $a$ and in particular under local supersymmetry transformations. The non-zero supersymmetry transformations are

$$
\begin{aligned}
\delta e^{a} & =\frac{1}{2} \bar{\zeta}^{i} \gamma^{a} \psi^{i} \\
\delta \psi^{i} & =\mathcal{D} \zeta^{i} \\
\delta C & =i \epsilon_{i j} \bar{\zeta}^{i} \psi^{j}
\end{aligned}
$$

where $\zeta^{i}$ are anticommuting spinor parameters. The field equations of (2.7) are equivalent to the vanishing of the components, (2.5), of the curvature two-form $F(a)$. We shall be interested in solutions of these (vacuum) equations for which the spacetime is asymptotically conical at spatial infinity, i.e. the metric is asymptotic to

$$
d s^{2}=d t^{2}-\lambda^{2} d r^{2}-r^{2} d \varphi^{2} \quad, \quad \lambda>1
$$

as $r \rightarrow \infty$. From the CS formulation of the theory, one can deduce [13] that the total mass relative to Minkowski spacetime is given by ${ }^{\star}$

$$
M=-\oint_{\infty}\left(\omega^{\underline{0}}-\bar{\omega}^{\underline{0}}\right)
$$

where $\bar{\omega}$ is the Minkowski spacetime spin connection. Note that the term involving $\bar{\omega}$ depends on the choice of frame and is not necessarily zero, as can easily be verified by choosing the natural frame of polar coordinates. From this definition one may

\footnotetext{
$\star$ Underlining indicates a frame index, i.e. $a=(\underline{0}, \underline{i})$, whereas $m=(0, i)$.
} 
verify the result of [16] that the mass of an asymptotically-conical spacetime equals the deficit angle, i.e.

$$
M=2 \pi\left(1-\frac{1}{\lambda}\right)
$$

We also require that the vector field $A$ be asymptotic to $A_{\varphi}^{\infty} d \varphi$, where $A_{\varphi}^{\infty}$ is a constant. The total $A$-charge can be defined by

$$
Q=\oint_{\infty} A=2 \pi A_{\varphi}^{\infty}
$$

In the case that the circle at infinity is contractible, all $U(1)$ gauge transformations, $A \rightarrow A^{\prime}=A+d \alpha$, with $d \alpha=\mathcal{O}(1 / r)$, are connected to the identity. In this case the charge $Q$ is clearly gauge-invariant. If, however, the circle at infinity is not contractible, as occurs for conical point-particle spacetimes, then there are additional 'large' gauge transformations, i.e. those not connected to the identity, and the charge $Q$ is not gauge invariant but can change by a multiple of $2 \pi$. In these circumstances, the charge $Q$ is well-defined only modulo $2 \pi$.

\section{Killing Spinors}

A bosonic configuration of $(2,0)$ Poincaré supergravity will preserve some of the supersymmetry if there is a non-zero $\zeta$ such that $\delta_{\zeta} \psi^{i}=0$. Since this equation is linear in the anticommuting spinor parameters $\zeta^{i}$ its consequences are unchanged if $\zeta^{i}$ is replaced by commuting spinors. It is also convenient to choose a complex basis for these commuting spinors. In such a basis, 'supersymmetric' bosonic configurations are those backgrounds for which the equation

$$
(D-i A) \kappa=0
$$

has non-trivial solutions for complex commuting spinor $\kappa$. The integrability con- 
dition for this equation is

$$
\left[F_{m n}^{a}(\omega) \gamma_{a}-2 F_{m n}(A)\right] \kappa=0
$$

This integrability condition is satisfied by all configurations that satisfy the vacuum equations. Although (3.2) is a necessary condition for the existence of solutions of (3.1) it is not sufficient; additional conditions must be satisfied in order for (3.1) to have non-trivial solutions for $\kappa$. To find these additional conditions, we first write the general solution of (3.1) for $\kappa$ at a point $p$, in terms of its value at a point $q$, as follows:

$$
\kappa(p)=\mathcal{P} \exp \left[\int_{C}\left(-i \frac{1}{2} \omega^{a} \gamma_{a}+i A\right)\right] \kappa(q)
$$

where $C$ is a path that interpolates between $q$ and $p$, and $\mathcal{P}$ indicates path-ordering of the exponential. This solution is not necessarily single-valued because it may depend on the path $C$. However, since the connections $\omega$ and $A$ are flat the solution can depend only on the homotopy class, $[C]$, of the path $C$. To compute the path dependence of the solution for $\kappa$, let $C^{\prime}$ be another path between $q$ and $p$. Then $\kappa$ is well-defined if and only if the condition

$$
\lambda([\Gamma]) \kappa(q)=\mathcal{P} \exp \left[\oint_{\Gamma}\left(-i \frac{1}{2} \omega^{a} \gamma_{a}+i A\right)\right] \kappa(q)
$$

is satisfied, where $\Gamma=\left(C^{\prime}\right)^{-1} \cdot C$ is a closed path from $q$ to $q$ and $\lambda([\Gamma])$ is a sign depending on the choice of spin structure. The choice of spin structure corresponds to the choice of periodic or anti-periodic boundary conditions for the spinors on the non-trivial fundamental homology cycles of space-time. The spin structure corresponding to periodic boundary conditions is called 'even', and the spin structure corresponding to antiperiodic boundary conditions is called 'odd'. The condition (3.4) is trivially satisfied if the spacetime is simply connected; the holonomies of both $A$ and $\omega$ are then equal to one. The interesting case is when the spacetime is not simply connected, in which case the existence of covariantly 
constant spinors depends on the holonomy of the flat connections $A$ and $\omega$ and also on the choice of spin structure. If only one of the connections $\omega$ and $A$ has non-trivial holonomy the equation (3.4) does not have solutions, and the vacuum solution will not be supersymmetric, so it remains to examine the case that both connections have non-trivial holonomy. The holonomy of $\omega$ evaluated at any closed path is an element of $S L(2, \mathbb{R})$. This holonomy is called elliptic, parabolic or hyperbolic depending on whether the value of the trace of its holonomy $2 \times 2$ matrix is less than, equal to, or larger than two, respectively. It is clear that in order to satisfy (3.4) the holonomy of $\omega$ must be elliptic. So, possibly after a gauge transformation, (3.4) can be rewritten as

$$
\lambda([\Gamma]) \kappa(q)=\mathcal{P} \exp \left[\oint_{\Gamma}\left(-i \frac{1}{2} \omega^{\underline{0}} \gamma_{\underline{0}}+i A\right)\right] \kappa(q) .
$$

Next, we pick $\kappa(q)$ to satisfy

$$
\gamma_{0} \kappa(q)= \pm \kappa(q)
$$

in which case the equation (3.5) becomes

$$
\lambda([\Gamma]) \kappa(q)=\mathcal{P} \exp \left[\oint_{\Gamma}\left(\mp i \frac{1}{2} \omega^{\underline{0}}+i A\right)\right] \kappa(q) .
$$

which is satisfied if and only if the holonomy of $\mp \frac{1}{2} \omega^{0}$ cancels the holonomy of $A$ up to a sign, depending on the choice of even or odd spin structure. We will refer to this as the 'holonomy condition'. In $2+1$ dimensions, the static multi pointparticle spacetime is flat everywhere except at isolated conical singularities. These spacetimes are non-simply connected if one removes the singular worldlines of the $K$ particles; space is then topologically a plane with $K$ punctures. The metric of 
such a spacetime is

$$
d s^{2}=d t^{2}-h^{2} \delta_{i j} d x^{i} d x^{j}, \quad i, j=1,2
$$

where

$$
h^{2}=\prod_{\ell=1}^{K}\left|x-x_{\ell}\right|^{-\frac{m_{\ell}}{\pi}}
$$

$m_{\ell}$ are the masses of the particles and $r_{\ell}$ are their positions. The only non-zero component of the connection $\omega$ is

$$
\omega_{i}^{0}=\epsilon^{j k} \delta_{k i} h^{-1} \partial_{j} h
$$

It can be shown using Cauchy's theorem that the holonomy of $\mp \frac{1}{2} \omega \underline{0}$ evaluated on a path $\Gamma_{n}$ that encloses $n \leq K$ particles with masses $m_{1}, \ldots, m_{n}$ is

$$
\exp \left[ \pm \frac{i}{2} M_{n}\right]
$$

where $M_{n}=\sum_{\ell=1}^{n} m_{\ell}$. There are $2^{K}$ different spin structures on this spacetime. If we define the charge $Q_{n}$ of the $n$ point particles by

$$
Q_{n}=\oint_{\Gamma_{n}} A
$$

then the holonomy condition implies that

$$
M_{n}=\mp 2 Q_{n}+2 \epsilon\left(\left[\Gamma_{n}\right]\right) \pi \nu
$$

where $\nu$ is an integer and $\epsilon\left(\left[\Gamma_{n}\right]\right)$ is equal either to 1 or to 2 , depending on whether the spin structure is chosen to be odd or even, respectively. Recalling that $Q_{n}$ is 
defined only $\bmod 2 \pi$, and that $M=M_{K}$ is restricted by $0 \leqslant M<2 \pi$, we see that for an even spin structure (3.13) is equivalent to

$$
M_{n}=2\left|Q_{n}\right|
$$

where we choose $Q_{n}$ such that $\left|Q_{n}\right|<\pi$. For an odd spin structure we can choose $\nu$ such that

$$
M_{n}=2\left|Q_{n} \pm \pi\right|
$$

where the sign must be chosen to ensure that $M<2 \pi$. In either case the relevant holonomy condition must hold for every closed path, and this implies that the mass of each particle is related to its charge as above. In particular, we find that the total mass and charge are related as in (1.9). Thus, the conditions on the masses and charges required for the existence of a Killing spinor depend on the choice of spin structure. We remark that the case of one particle with mass $M=\pi$ and charge $Q=\mp \frac{1}{2} \pi$ is special in that the corresponding spacetime admits Killing spinors of either spin structure. We conclude this section with a remark. First, note that a spinor that is Killing in some region of spacetime, e.g. an asymptotic region, cannot necessarily be extended to a Killing spinor of the entire spacetime. This is because the holonomy matching condition should hold for every fundamental homology cycle of spacetime.

\section{Matter coupling to $(2,0)$ supergravity}

We shall now consider the coupling of the general $\mathrm{N}=2$ sigma model to $(2,0)$ Poincaré supergravity. As explained in the introduction, the off-shell supermultiplet includes the gauge field one-forms $A$ and $C$ and an auxiliary scalar field $K$ in addition to the dreibein one-form $e^{a}$ and the two Majorana gravitini, which we 
may exchange for a single Dirac spinor one-form $\psi$. The Lagrangian is

$$
\mathcal{L}_{\text {grav }}=-e R+i \varepsilon^{m n p} \bar{\psi}_{m} \hat{\mathcal{D}}_{n} \psi_{p}-2 \varepsilon^{m n p} C_{m} \partial_{n} A_{p}+2 e K^{2}
$$

where

$$
\hat{\mathcal{D}}_{m}=\mathcal{D}_{m}-\frac{1}{4} e^{-1} \varepsilon_{m n p} \gamma^{n} V^{p}+\frac{i}{2} \gamma_{m} K
$$

and the Dirac conjugate is defined by $\bar{\psi}=\psi^{\dagger}\left(i \gamma^{\underline{0}}\right)$, which is equivalent to the previous definition if $\psi$ is real. This Lagrangian is invariant, up to a total derivative, under the following supersymmetry transformations

$$
\begin{aligned}
\delta e_{m}{ }^{a} & =\frac{1}{4}\left(\bar{\epsilon} \gamma^{a} \psi_{m}-\bar{\psi}_{m} \gamma^{a} \epsilon\right) \\
\delta \psi_{m} & =\hat{\mathcal{D}}_{m} \epsilon \\
\delta C_{m} & =-\frac{1}{2} \bar{\psi}_{m} \epsilon+\frac{1}{2} \bar{\epsilon} \psi_{m} \\
\delta A_{m} & =\frac{i}{8} e^{-1} \varepsilon_{m n p} \epsilon \gamma^{n} \chi^{p}+c . c . \\
\delta K & =-\frac{1}{8} \bar{\epsilon} \gamma \cdot \chi+c . c .
\end{aligned}
$$

where

$$
\begin{aligned}
V^{m} & \equiv e^{-1} \varepsilon^{m n p} \partial_{n} C_{p} \\
\chi^{m} & \equiv e^{-1} \varepsilon^{m n p} \mathcal{D}_{n} \psi_{p}
\end{aligned}
$$

Note that the Lagrangian (4.1) is equivalent (for $\mu=-1$ ) to that of (2.7) after elimination of the auxiliary field $K$ by its algebraic equation of motion. Note also that the supersymmetry transformations (4.3) differ from the CS supersymmetry transformations by the addition of transformations proportional to the field equations. The transformations given here are the 'geometrical' ones. Since these transformations close to give general coordinate and other gauge tranformations without the use of the field equations, the coupling to matter will not change them. The three-dimensional off-shell $\mathrm{N}=2$ scalar multiplet has the field content $(\Phi, \lambda, F)$ where $\Phi$ is a physical complex scalar, $\lambda$ a Dirac spinor and $F$ a complex auxiliary 
field. The coupling of $(2,0)$ supergravity to $\mathrm{n}$ such multiplets leads to a $(2,0)$ locally supersymmetric sigma-model with an $n$ complex-dimensional Kähler target space. One method of constructing this model would be through the development of a tensor calculus, which is effectively equivalent to the superfield method developed in section 6. Here we shall make use of the order-by-order 'Noether method'; since the matter auxiliary fields play no role in this method they will be omitted from the start. It is also convenient to introduce $2 n$ real scalar fields $\phi^{I}$, instead of $n$ complex scalar fields. Thus, the matter fields that we shall need to consider are

$$
\left(\phi^{I}, I=1 \ldots 2 n ; \lambda^{A}, \bar{\lambda}_{A}, A=1 \ldots n\right)
$$

where

$$
\bar{\lambda}_{A}=\left(\lambda^{A}\right)^{\dagger}\left(i \gamma^{\underline{0}}\right)
$$

With this notation it is necessary to introduce a target space vielbein $\left(f_{I}{ }^{A}, f_{I A}=\right.$ $\left.\overline{\left(f_{I}^{A}\right)}\right)$ and its inverse $\left(f^{I}{ }_{A}, f^{I A}=\overline{\left(f_{A}^{I}\right)}\right)$ satisfying

$$
\begin{aligned}
& f^{I}{ }_{A} f_{I}{ }^{B}=\delta_{A}^{B} \quad f^{I A} f_{I B}=\delta_{B}^{A} \\
& f^{I}{ }_{A} f_{I B}=0 \quad f^{I A} f_{I} B=0 \\
& f^{I}{ }_{A} f_{J}{ }^{A}+f^{I A} f_{J A}=\delta_{J}^{I} .
\end{aligned}
$$

The Kähler two-form $\Omega$ can be defined via the relation

$$
g_{I J}-i \Omega_{I J}=2 f_{I}^{A} f_{J A}
$$

where $e=\operatorname{det} e_{m}{ }^{a}$ and $g$ is the target space metric. We refer to [13] for more details of the notation. The locally $(2,0)$-supersymmetric sigma-model Lagrangian for fields (4.5) is

$$
\begin{aligned}
\mathcal{L}_{\sigma}= & \frac{1}{2} e\left[g_{I J} \partial_{m} \phi^{I} \partial^{m} \phi^{J}-\bar{\lambda}_{A} \gamma^{m} \nabla_{m} \lambda^{A}\right]+\frac{1}{2} C_{m} j_{t o p}^{m} \\
& -\frac{i}{2} e\left[\bar{\lambda}_{A} \gamma^{m} \gamma^{n} \psi_{m} f_{I}^{A}+\bar{\psi}_{m} \gamma^{n} \gamma^{m} \lambda^{A} f_{I A}\right] \partial_{n} \phi^{I}+\text { (quartic fermion terms) }
\end{aligned}
$$

where $\nabla=\mathcal{D}+L$, with $L$ equal to the spin-connection of the Levi-Civita connection 
with respect to the frame $f$. Notice that the central charge gauge potential $C$ couples to the topological current

$$
j_{\text {top }}^{m} \equiv \frac{1}{2} \varepsilon^{m n p} \Omega_{I J} \partial_{n} \phi^{I} \partial_{p} \phi^{J} .
$$

We have not made any attempt to calculate the quartic fermion terms because they are not important for our present purposes. The complete construction, using superfield methods, will be presented in section 6. The action (4.9) is invariant under the transformations of the supergravity fields given above together with the following supersymmetry transformations of the matter fields:

$$
\begin{aligned}
\delta \phi^{I} & =\frac{i}{2} f^{I}{ }_{A} \bar{\epsilon} \lambda^{A}+\frac{i}{2} f^{I A} \bar{\lambda}_{A} \epsilon \\
\delta \lambda^{A} & =-\frac{i}{2} f_{I}{ }^{A} \gamma^{m} \epsilon \partial_{m} \phi^{I}+(\text { cubic fermion terms) } .
\end{aligned}
$$

The Lagrangian for the coupling of $(2,0)$ Poincaré supergravity to $\mathrm{N}=2$ sigmamodel matter is simply the sum $\mathcal{L}=\mathcal{L}_{\text {grav }}+\mathcal{L}_{\text {sigma }}$ from which the supergravity auxiliary field $K$ may be trivially eliminated (neglecting possible additional quartic fermion terms) to arrive at the Lagrangian (in a condensed notation)

$$
\begin{array}{r}
\mathcal{L}=e\left[-R+\frac{1}{2}(\partial \phi)^{2}-\bar{\lambda} \gamma \cdot \nabla \lambda-\left\{\frac{i}{2} \partial_{n} \phi \cdot \bar{\lambda} \gamma^{m} \gamma^{n} \psi_{m}+c . c .\right\}\right] \\
+i \varepsilon^{m n p} \bar{\psi}_{m} \mathcal{D}_{n} \psi_{p}-2 \varepsilon^{m n p} C_{m} \partial_{n} A_{p}+\frac{1}{2} C_{m} j_{\text {top }}^{m}
\end{array}
$$

For what follows we shall need only the bosonic field equations of this Lagrangian, and the fermion transformation laws, in backgrounds for which the fermion fields vanish. The bosonic field equations are

$$
\begin{aligned}
\varepsilon^{m n p} \partial_{n} A_{p} & =\frac{1}{4} j_{t o p}^{m} \\
G_{m n} & =\frac{1}{2} T_{m n} \\
V^{m} & =0 \\
\nabla^{2} \phi & =0 .
\end{aligned}
$$


where $T_{m n}$ is the energy-momentum tensor

$$
T_{m n}=\partial_{m} \phi \cdot \partial_{n} \phi-\frac{1}{2} g_{m n} \partial \phi \cdot \partial \phi
$$

The first of these equations states that the two-form field strength, $F=d A$, is proportional to the pullback of the Kähler two-form, $\Omega$, of the target space. Since the automorphism group is $U(1)$ (rather than $\mathbb{R}$ ), as a consequence of the fermion couplings, the two-form $F$ is an integral class in the cohomology of spacetime, and hence so is the pullback of $\Omega$. This imposes a condition on the target manifolds for which the model is consistent. We note here that this condition is clearly satisfied by Hodge manifolds, for which the Kähler form is an integral class in the cohomology. This is very similar to the restriction on the target spaces of $\mathrm{D}=3+1$ sigma-models coupled to $\mathrm{N}=1$ supergravity [19]. The fermion supersymmetry transformations in bosonic, on-shell, backgrounds are

$$
\begin{aligned}
\delta \psi_{m} & =\mathcal{D}_{m} \epsilon \\
\delta \lambda^{A} & =-\frac{i}{2} f_{I}^{A} \gamma^{m} \epsilon \partial_{m} \phi^{I}
\end{aligned}
$$

Note that the Killing spinor condition $\delta \psi_{m}=0$ is unaffected by the presence of matter. However, its integrability condition (3.2) is no longer satisfied by all solutions of the field equations. In fact, using the field equations (4.13) we find that the integrability condition becomes

$$
\left[\gamma^{n} T_{n}^{m}-j_{\text {top }}^{m}\right] \chi=0
$$

which can be rewritten as

$$
g^{I J}\left(P_{I K} \gamma^{n} \partial_{n} \phi^{K}\right) \gamma^{m}\left(P_{J L} \gamma^{p} \partial_{p} \phi^{L}\right) \chi=0
$$

where $P$ is the projector

$$
P_{I J}=g_{I J}-i \Omega_{I J} .
$$


By contracting (4.17) with $\bar{\chi}$ we deduce that

$$
g^{I J} \xi_{I} \gamma^{m} \xi_{J}=0
$$

where

$$
\xi_{I} \equiv P_{I J} \gamma^{n} \partial_{n} \phi^{J} \chi
$$

The left hand side of (4.19) is manifestly timelike, and hence non-zero, unless $\xi_{I}=0$. Thus, the integrability condition for a Killing spinor in the presence of matter is equivalent to

$$
\xi_{I}=0
$$

For Killing spinors satisfying (3.6), this condition is satisfied by matter fields for which

$$
\underline{e}_{\underline{0}}^{m} \partial_{m} \phi^{I}=0, \quad P_{I J}\left(\underline{e}^{m} \pm i \underline{e}^{m}\right) \partial_{m} \phi^{J}=0
$$

Choosing complex coordinates $\phi^{\alpha}$ on the target space, with complex conjugates $\bar{\phi}^{\bar{\alpha}}$, these equations become

$$
e_{\underline{0}}^{m} \partial_{m} \phi^{\alpha}=0, \quad e_{ \pm}^{m} \partial_{m} \phi^{\alpha}
$$

where $e_{ \pm}^{m}=e_{\underline{1}}^{m} \pm i e_{\underline{2}}^{m}$. For the static spacetime metric

$$
d s^{2}=d t^{2}-e^{-\sigma(z, \bar{z})} d z d \bar{z}
$$

the first of eqs. (4.23) is trivially satisfied by time-independent matter fields while the second is satisfied by holomorphic functions, i.e

$$
\phi^{\alpha}=\phi^{\alpha}(z)
$$

The Einstein equation then implies that

$$
\partial \bar{\partial}[\sigma(z, \bar{z})-\mathcal{K}(\phi(z), \bar{\phi}(\bar{z}))]=0
$$

where $\mathcal{K}(\phi, \bar{\phi})$ is the Kähler potential of the target space metric. Similar equations have been considered previously, and solutions to them discussed, in the context 
of cosmic string solutions of $3+1$ dimensional field theories [20,21]. We have now shown, in the context of the $(2,0)$ Poincaré supergravity, that solutions of the form (4.24) and (4.25) satisfying (4.26) are supersymmetric. We expect that this is also true for the corresponding cosmic string solutions in $3+1$ dimensions.

\section{An energy theorem}

A customary feature of spacetimes admitting Killing spinors is that they saturate a Bogomol'nyi-type bound on the energy. To investigate this possibility here we proceed as usual by defining the Nester tensor

$$
E^{m n}=-\frac{i}{2} \bar{\chi} \gamma^{m n p} \mathcal{D}_{p} \chi+\text { c.c. }
$$

Given a non-singular Cauchy surface $\Sigma$, we consider the integral $\int_{\Sigma} d S_{m} D_{n} E^{m n}$. Using the field equations, we obtain

$$
\int_{\Sigma} d S_{m} D_{n} E^{m n}=-\int_{\Sigma} d S_{m}\left[\frac{i}{2} \overline{\mathcal{D}_{n} \chi} \gamma^{m n p} \mathcal{D}_{p} \chi+\text { c.c. }\right]+\int_{\Sigma} d S_{m} K^{m}
$$

where

$$
K^{m}=-\frac{i}{2} G^{m}{ }_{n} \bar{\chi} \gamma^{n} \chi-i e^{-1} \varepsilon^{m n p} \partial_{n} A_{p} \bar{\chi} \chi
$$

Consider first the left hand side of (5.2). We may choose the spacelike surface $\Sigma$ to be a surface of constant $t$, so that the only non-zero component of $d S_{m}$ is $d S_{0}$. Then

$$
\int_{\Sigma} d S_{m} D_{n} E^{m n}=\oint d \varphi \int d r\left[\partial_{r}\left(\bar{\chi} \mathcal{D}_{\varphi} \chi\right)-\partial_{\varphi}\left(\bar{\chi} \mathcal{D}_{r} \chi\right)\right]
$$

The first term in the integral on the right hand side of this equation equals

$$
\oint_{\infty} d \varphi \bar{\chi} \mathcal{D}_{\varphi} \chi=\oint_{\infty} d \varphi\left(\bar{\chi} \bar{D}_{\varphi} \chi+\frac{i}{2} \bar{\chi}\left(\omega_{\varphi}^{a}-\bar{\omega}_{\varphi}^{a}\right) \gamma_{a} \chi-i A_{\varphi}^{\infty} \bar{\chi} \chi\right)
$$

In order for this to be a combination of the mass and the charge, as defined in (2.10) and (2.12), we require $\chi \sim \chi_{\infty}$ as $r \rightarrow \infty$, where $\chi_{\infty}=e^{i f(\phi)} \chi_{0}$ with $\chi_{0}$ 
a non-zero constant spinor, and that $\omega_{\varphi}^{a} \rightarrow 0$, for $a \neq \underline{0}$. The second term in the integral on the right hand side of (5.4) is zero if it is convergent. Convergence requires that $\int d r \bar{\chi} \mathcal{D}_{r} \chi<\infty$, which means that $\mathcal{D}_{r} \chi$ must go to zero faster than $1 / r$ as $r \rightarrow \infty$. These conditions on $\omega$ and $\chi$ are guaranteed if the metric is of the form $g_{m n}=g_{m n}^{\infty}+h_{m n}$, where $g_{m n}^{\infty}$ is the metric given in (2.9), and $h_{m n}$ has the properties

$$
\lim _{r \rightarrow \infty} h_{m n}=0 \quad, \quad \lim _{r \rightarrow \infty}\left(r h_{m n, p}\right)=0 .
$$

Then, if we use the fact that $\bar{\chi}=i \chi^{\dagger} \gamma^{0}$, and take

$$
\chi_{\infty}^{\dagger} \chi_{\infty}=1, \quad \gamma^{\underline{0}} \chi_{\infty}= \pm \chi_{\infty},
$$

the right hand side of (5.5) reduces to $\frac{1}{2}(M \pm 2 Q)+\oint_{\infty} d \varphi \bar{\chi}_{\infty} \bar{D}_{\varphi} \chi_{\infty}$, and we therefore deduce that

$$
\int_{\Sigma} d S_{m} D_{n} E^{m n}=\frac{1}{2}(M \pm 2 Q)+\oint_{\infty} d \varphi \bar{\chi}_{\infty} \bar{D}_{\varphi} \chi_{\infty}
$$

Consider now the right hand side of (5.2). Provided that the vector $K$ is futuredirected timelike or zero, the second integral is non-negative. This condition is satisfied for the supersymmetric sigma-model matter of the previous section. This can be seen, following the discussion in [13] of the adS case, by using the field equations (4.13) to rewrite $K^{m}$ as

$$
\begin{aligned}
K^{m} & =-\frac{i}{4} \bar{\chi}\left(T_{n}^{m} \gamma^{n}-j_{t o p}^{m}\right) \chi \\
& =-\frac{i}{4} g^{I J} \xi_{I} \gamma^{m} \xi_{j},
\end{aligned}
$$

where $\xi_{I}$ was defined in (4.20). The right hand side is a manifestly future-directed timelike vector field, as required. Thus, if we can establish that the first integral is also non-negative we will have established a bound on the mass $M$. The first 
integral is indeed non-negative if the spinor $\chi$ is chosen to satisfy the $2+1$ analogue of the Witten condition,

$$
{ }^{(2)} g^{i j} \gamma_{i} \mathcal{D}_{j} \chi=0
$$

where ${ }^{(2)} g^{i j}$ is the inverse of the spatial part of the metric. It turns out, however, that the only non-zero solutions of (5.10) with the required boundary conditions are Killing spinors, so that instead of the expected inequality we find an equality. We now explain this point. From the asymptotic conditions (5.6) it is easy to see that

$$
\begin{aligned}
& \lim _{r \rightarrow \infty} r^{2} g^{r \varphi}=0 \quad, \quad \lim _{r \rightarrow \infty} g^{r r}=-\frac{1}{\lambda^{2}} \quad, \quad \lim _{r \rightarrow \infty} r^{2} g^{\varphi \varphi}=-1, \\
& \lim _{r \rightarrow \infty} \gamma_{r}=\lambda \gamma_{1} \quad, \quad \lim _{r \rightarrow \infty} \frac{1}{r} \gamma_{\varphi}=\gamma_{\underline{2}}, \\
& \lim _{r \rightarrow \infty} \mathcal{D}_{\varphi} \chi=\left[\partial_{\varphi} \pm \frac{1}{2}\left(1-\frac{M}{2 \pi}\right)-\frac{Q}{2 \pi}\right] \chi_{\infty} \quad, \quad \lim _{r \rightarrow \infty} r \mathcal{D}_{r} \chi=0 .
\end{aligned}
$$

Using this, it is clear that

$$
0=\lim _{r \rightarrow \infty}{ }^{(2)} g^{i j} \gamma_{i} \mathcal{D}_{j} \chi=-\gamma_{\underline{2}}\left[\partial_{\varphi} \pm \frac{1}{2}\left(1-\frac{M}{2 \pi}\right)-\frac{Q}{2 \pi}\right] \chi_{\infty}
$$

This last equation tells us that $\chi_{\infty}$ is a Killing spinor of a conical spacetime of charge $Q$ and mass $M$. We have shown earlier that such spinors exist only if either $M=2|Q|$ or $M=2|Q \pm \pi|$. Moreover, (5.12) implies that the right hand side of (5.8) vanishes which in turn implies that the spinor $\chi$ is a Killing spinor of the spacetime and not merely asymptotic to one. Thus, the Witten-Nester method applied to 2+1 Poincaré gravity leads to an equality rather than a bound! In retrospect this result should not be so surprising because, in distinction to the adS case [13], the mass is determined by the leading terms in the asymptotic metric, i.e. by the boundary conditions. Note that this argument is independent of the presence of matter. Indeed, as noted in the previous section, the existence of a Killing spinor for a solution of the field equations requires that $\xi_{I}=0$, which 
implies that $K^{m}=0$. We conclude this section with a comment on the role of angular momentum. A spacetime of the form [16]

$$
d s^{2}=\left(d t+\frac{J}{2 \pi} d \varphi\right)^{2}-\lambda^{2} d r^{2}+d \varphi^{2},
$$

where $J$ is the angular momentum, has exactly the same spin connection as (2.9), so the Killing spinor equation is not altered. The argument leading to (5.12) is not modified if $g_{m n}^{\infty}$ is taken to be (5.13) so we arrive at the same conclusion as before. In other words, in contrast to the adS case, angular momentum is irrelevant.

\section{Off-shell superfields}

As in four dimensions, it is convenient to discuss off-shell superspace supergravity in three-dimensional spacetime starting from a superconformal perspective. One possible definition of a superconformal structure on $N$-extended superspace (the $N=2$ case was discussed in [7]) is as follows [22]: a superconformal structure on $(3 \mid 2 N)$ dimensional superspace $M$ is a choice of odd tangent bundle $F$ (of rank $(0 \mid 2 N))$ and a reduction of the structure group of the frame bundle of $F$ to $G:=G L^{+}(2, R) \times{ }_{Z_{2}} O(N)$ such that the Frobenius tensor of $F$ coincides with the natural tensor associated with $G$. The Frobenius tensor of the sub-bundle $F$ of the tangent bundle $T$ is defined by computing the commutators of vector fields which are sections of $F$ and evaluating them modulo $F$. This defines a tensor field taking its values in $\Lambda^{2} F^{*} \otimes B$ where $B=T / F$. The choice of $G$ means that, locally at least, $F=S \otimes V$ where $S$ has rank $(0 \mid 2)$ and $V$ has rank $(N \mid 0)$. The natural tensor associated with $G$ is the product of the metric on $V$ with the Dirac matrices (considered as defining a map from from $\Lambda^{2} S$ to $B$ ). If we let $E_{\alpha i}$ denote a local basis of $F$ and $E^{a}$ a local basis of $B^{*}$, then these bases can be chosen such that the components of the Frobenius tensor are given by

$$
<\left[E_{\alpha i}, E_{\beta j}\right], E^{c}>=\delta_{i j}\left(\gamma^{c}\right)_{\alpha \beta}
$$

where $<>$ denotes the pairing between vectors and forms. In order to unravel the consequences of this structure it is convenient to make a choice of $B$ as a 
subbundle of $T$ and to introduce a connection which we choose to take values in $s l(2, \mathbb{R}) \oplus o(N)$. These choices can be made in such a way that the components of the torsion tensor are, at dimension zero ${ }^{\star}$,

$$
T_{\alpha i \beta j}{ }^{c}=-\delta_{i j}\left(\gamma^{c}\right)_{\alpha \beta}
$$

(so that this part of the torsion is identified with the Frobenius tensor); at dimension one-half,

$$
T_{\alpha i b}{ }^{c}=T_{\alpha i \beta j}{ }^{\gamma k}=0
$$

at dimension one,

$$
T_{a b}^{c}=0
$$

and

$$
T_{a \beta j}{ }^{\gamma k}=i\left(\gamma^{b}\right)_{\beta}^{\gamma} G_{a b j}{ }^{k}-i\left(\gamma_{a}\right)_{\beta}^{\gamma} K_{j}^{k}
$$

while at dimension three-halves the leading component of the torsion in a $\theta$ expansion is essentially the field strength tensor of the gravitini. The tensor $G$ is antisymmetric on both its internal and Lorentz indices while $K$ is symmetric. The components of the curvature tensor can be computed using the Bianchi identities, and for general $N$ one finds that the geometry is described by the two superfields $G$ and $K$ together with a third, which for $N \geqslant 4$ is a dimension one scalar $A_{i j k l}$ totally antisymmetric on its internal indices. The component fields corresponding to this structure divide into a conformal supergravity (CSG) multiplet and a compensating Weyl multiplet which is an entire scalar superfield. The CSG multiplet is

$$
e_{m}{ }^{a} ; \psi_{m i} ; A_{m i j}, A_{i j k l} ; \rho_{i j k}, \sigma_{i j k l m} ; B_{i j k l}, \ldots
$$

where each field is antisymmetric on its internal indices. The leading components of the fields $K$ and $G$ above belong to the Weyl multiplet. There are two special

\footnotetext{
$\star$ This corresponds to a rescaling of the generator $P_{a}$ in the supersymmetry algebra of (1.1).
} 
cases, $N=4$ and $N=8$. In $N=4$ one can reduce the internal symmetry group from $O(4)$ to $S O(3)$, in which case the CSG multiplet is simply $\left(g_{m n}, \psi_{m i}, A_{m i j}\right)$ where the gauge field $A_{m i j}$ is now self-dual on its internal indices. In $N=8$ one can impose self-duality on the scalar field $A_{i j k l}$. The first few components of a scalar superfield are

$$
A ; \lambda_{i} ; K_{i j}, G_{a i j} ; \chi_{a i j k} \ldots
$$

where $G$ and $\chi$ are antisymmetric on their internal indices, $K$ is symmetric and $\chi$ is gamma-traceless. We begin the discussion of Poincaré supergravity with $N=2$. The compensating multiplet can be constrained to be chiral, by taking $K_{i j}=\tilde{K}_{i j}$ (tracefree), or to be real linear by taking $K_{i j}=\delta_{i j} K$. In the former case the field $G_{a}=\partial_{a} B$, at least at the linearised level, while in the latter case $G_{a}$ is conserved. At the linearised level these multiplets are constructed from a general real scalar superfield by imposing the constraints

$$
D^{2} A=0
$$

or

$$
\tilde{D}^{i j} A=0
$$

respectively, where $D^{2}$ and $\tilde{D}^{i j}$ are defined by

$$
D_{\alpha i} D_{\beta j}=\epsilon_{\alpha \beta} \epsilon_{i j} D^{2}+\epsilon_{\alpha \beta} \tilde{D}^{i j}+\frac{1}{2} \delta_{i j}\left(\gamma^{a}\right)_{\alpha \beta} \partial_{a} .
$$

The chiral compensator corresponds to type $(1,1)$ Poincaré supergravity. The fields $A, B$ and $\lambda$ get absorbed by the metric, gravitino and $S O(2)$ gauge field respectively to yield the following multiplet:

$$
e_{m}{ }^{a} ; \psi_{m i} ; A_{m}, \tilde{K}_{i j}
$$

To implement this in superspace it is more convenient to reduce the structure group to $S L(2, \mathbb{R})$ and to combine the Weyl and $S O(2)$ parameters into a complex 
scalar superfield which can then be constrained to be chiral. The corresponding constraints resemble the $N=1$ minimal constraints in four dimensions and can be solved in the Ogievetsky-Sokatchev formalism [23]; alternatively, one can observe that the action is $\int_{M} E$ where $E$ is the superdeterminant of the supervielbein, and derive the equations of motion by solving the constraints for a deformation of the supervielbein [24]. The equations of motion imply that the superspace is flat, as expected. In the $(2,0)$ case, with $K_{i j}=\delta_{i j} K$, the Poincaré supermultiplet that is obtained by combining the conformal SG multiplet with the compensator is

$$
e_{m}{ }^{a}, C_{m} ; \psi_{m i} ; A_{m}, K
$$

where $C_{m}$ is a gauge field whose field strength is $G$ and $A_{m}$ is the $S O(2)$ gauge field. This theory resembles closely new minimal supergravity in four dimensions [9] and is best described in superspace by introducing an abelian gauge field $C$ with field strength $G=d C$. The components of $G$ are, at dimension zero,

$$
G_{\alpha i \beta j}=-i \epsilon_{\alpha \beta} \epsilon_{i j}
$$

at dimension one-half,

$$
G_{a \beta j}=0
$$

while at dimension one we find $G_{a b}$ which is identified with the field occuring in the torsion. Again the constraints can be solved in Ogievetsky-Sokatchev fashion, this time in a superspace with an extra bosonic coordinate to accommodate $C$. Alternatively one can follow [10]. In this approach one observes that there is a choice of gauge in which the spinorial part of the $S O(2)$ gauge field takes the form

$$
A_{\alpha+}=i D_{\alpha+} V
$$

where $V$ is a real prepotential and $D_{\alpha+}=\frac{1}{\sqrt{2}}\left(D_{\alpha 1}-i D_{\alpha 2}\right)$. The action is $\int_{M} E V$. The corresponding component action differs from the CS one (2.7) only by the 
addition of a term $K^{2}$. We now turn to the more interesting case of $N=3$. We first consider $(2,1)$ supergravity. The internal part of the structure group is to be reduced from $S O(3)$ to $S O(2)$, and so the compensating multiplet involves a total of three scalar superfields corresponding to the Weyl and $O(3) / O(2)$ parameters. This triplet of fields can be constrained to form a vector multiplet, which has components

$$
A, A_{i}, C_{m} ; \lambda, \lambda_{i}, \lambda^{\prime} ; K, K_{i}
$$

where $i=1,2$. Combining this multiplet with the CSG multiplet we find the following $(2,1)$ Poincaré supermultiplet

$$
e_{m}{ }^{a}, C_{m} ; \psi_{m i}, \psi_{m} \lambda ; K, K_{i}, A_{m}, A_{m i} ; \rho
$$

where $A_{m}$ is the automorphism gauge field and the remaining dimension one vectors are non-gauge auxiliaries. We have not constructed the superspace action for this theory, but it should be possible to construct one using harmonic superspace techniques [26]. The off-shell component action differs from the CS action by the addition of terms quadratic in the bosonic auxiliaries $A_{m i}, K, K_{i}$, and a term of the form $\lambda \rho$ which takes care of the auxiliary fermions. The $(3,0)$ theory is more complicated. In this case we have only the Weyl compensator at our disposal. If we impose $K_{i j}=\delta_{i j} K$, as in the $(2,0)$ case but now $i, j=1,2,3$, we find the following multiplet:

$$
A ; \lambda_{i} ; G_{a i} ; \chi_{a}
$$

where $G_{a i}$ is conserved and $\chi_{a}$ is gamma-traceless and conserved. On the other hand the conformal multiplet is

$$
e_{m}{ }^{a} ; \psi_{m i} ; A_{m i} ; \rho
$$

The problem lies with the dimension three-halves fields $\rho$ and $\chi$. It seems plausible that these should be combined into a conserved, but not gamma-traceless field $\Sigma_{a}$, 
so that, at the linearised level at least,

$$
\Sigma_{a}=\epsilon_{a b c} \partial^{b} \Lambda^{c}
$$

The field $\Lambda$ is a new gravitino. This suggests that the off-shell $(3,0)$ theory is in fact an $N=4$ theory with component field content

$$
e_{m}{ }^{a}, C_{m i j} ; \psi_{m i} ; A_{m i j}, K
$$

where now $i, j=1, . ., 4$ and both $C$ and $A$ are self-dual on their $S O(4)$ indices. This hypothesis can be tested by constructing an appropriate $N=4$ supergeometry, but this is not entirely straightforward owing to the fact that the central charge acts non-trivially off-shell. However, it appears that this can be done, although we have neither verified it completely nor constructed a superspace action. In outline, the construction starts with a superspace $M^{\prime}$ of dimension $(3+3 \mid 8)$ which can be thought of as some sort of affine bundle over $N=4$ superspace $M$. The structure group of $M^{\prime}$ geometry is taken to be $S L(2, \mathbb{R}) \times S O(3)$. A preferred set of coframes is denoted by $E^{\underline{A}}=\left(E^{A}, E^{I}\right)=\left(E^{a}, E^{\alpha i}, E^{I}\right)$, where $i=1, . ., 4$ and $I=1,2,3$. The connection, $\Gamma_{\underline{A}} \underline{B}$ has non-vanishing components $\Gamma_{a}^{b}, \Gamma_{\alpha i}{ }^{\beta j}=\delta_{\alpha}{ }^{\beta} A_{i}{ }^{j}+\delta_{i}{ }^{j} \Gamma_{\alpha}{ }^{\beta}$ and $\Gamma_{I}^{J}$, where

$$
\Gamma_{\alpha \beta}=-\frac{1}{4}\left(\gamma^{a b}\right)_{\alpha \beta} \Gamma_{a b}
$$

and

$$
\begin{gathered}
\Gamma_{I J}=\epsilon_{I J}{ }^{K} A_{K} \\
A_{i j}=f_{i j}{ }^{K} A_{K}
\end{gathered}
$$

with $f_{i j}{ }^{K}$ denoting the numerically invariant self-dual tensor. The non-vanishing components of the torsion are, at dimension zero,

$$
\begin{gathered}
T_{\alpha i \beta j}{ }^{c}=-\delta_{i j}\left(\gamma^{c}\right)_{\alpha \beta} \\
T_{\alpha i \beta j}{ }^{K}=-i \epsilon_{\alpha \beta} f_{i j}{ }^{K},
\end{gathered}
$$


and at dimension one,

$$
\begin{aligned}
T_{a \beta j}{ }^{\gamma k} & =-i\left(\gamma_{a}\right)_{\beta}{ }^{\gamma} \delta_{j}{ }^{k} K+\frac{i}{2}\left(\gamma^{b}\right)_{\beta}{ }^{\gamma} G_{a b j}{ }^{k} \\
T_{I \beta j}{ }^{\gamma k} & =\frac{2}{3} \epsilon_{\beta \gamma} f_{j k I} K+\frac{1}{4} f_{j k}{ }^{J} \epsilon_{I J K} G_{\beta \gamma}{ }^{K} \\
T_{a b}{ }^{K} & =G_{a b}{ }^{K} \\
T_{I J}{ }^{c} & =\frac{1}{2} \epsilon_{I J K} G^{c K} .
\end{aligned}
$$

This set of constraints is consistent with the Bianchi identities up to dimension one, which is as far as we have checked. We are encouraged to believe that it is fully consistent as the dimension one identities are non-trivial. For higher $N$ it seems to be more difficult to construct off-shell superspace formalisms which can be used to write down actions. This is because the multiplets for general $N$ contain high spin, high dimension, fields and involve covariant conservation conditions. One might think that it would be possible to mimic the CS formalism in superspace, but again one runs into difficulties for higher $N$. In this approach one introduces a superspace gauge potential $\mathcal{A}$ which takes its values in the (p,q) Poincaré superalgebra. Because this includes supertranslations, the correct equations of motion are simply $\mathcal{F}\left(=d \mathcal{A}+\mathcal{A}^{2}\right)=0$. However, because of high spin component fields it is difficult to construct an action which would lead to this equation for all $N$. In fact this is true even for ordinary supersymmetric Yang-Mills for which the conventional superspace approach works only for $N \leqslant 3[8]$ and the harmonic approach for $N$ up to 6 [25]. It is not ruled out that superspace actions exist for all $N$, but, as yet, it is not clear how to construct them. We conclude this section with a brief discussion of matter coupling in $(2,0)$ supergravity in the superspace formalism. To do this it is convenient to begin again with $N=2$ superconformal geometry. This may be reformulated in a way which emphasises its close affinity with Kähler geometry as follows: one has a real supermanifold $M$ of dimension (3|4) with a choice of odd tangent bundle, $F$, such that $F$ is equipped with a fibre complex structure, $I \in \Gamma(E n d F), I^{2}=-1$, and such that the Frobenius tensor, regarded as a $B$-valued form in $\wedge^{2} F^{*}$ is of type $(1,1)$ with respect to $I$. One can then verify 
that such a supermanifold is in fact a $C R$ supermanifold, which is to say that, if $F_{c}=\mathcal{F} \oplus \overline{\mathcal{F}}$, where $F_{c}$ is the complexification of $F$, then $\overline{\mathcal{F}}$ is involutive. This allows one to define a $C R$ exterior derivative, $\bar{D}$, satisfying $\bar{D}^{2}=0$. The structure group associated with the above superconformal structure is $S L(2, R) \cdot U(1) \times R^{*}$; it can be reduced to $S L(2, R) \cdot U(1)$ by the introduction of a fibre metric $g_{F} \in \Gamma\left(S^{2} F^{*}\right)$, which can be taken to be hermitian. This then allows one to define a fermionic Kähler 2-form $\omega_{F} \in \Gamma\left(\wedge^{2} F *\right)$ by lowering the contravariant index on $I$ using the fibre metric. The two-form $\omega_{F}$ is in fact a $(1,1)$ form and is furthermore $\bar{D}$-closed. It extends to a closed two-form on the whole space if and only if the superfield $K_{i j}=\delta_{i j} K$. The two-form obtained in this way is precisely the two-form $G$ introduced earlier. The matter field $\phi$ is introduced as a $C R$ map $\phi: M \rightarrow N$, where $N$ is the target space which is taken to be Kähler, that is $\phi$ is complex and chiral, $\bar{D} \phi=0$. The action for $(2,0)$ supergravity coupled to matter is then given by

$$
S=\int_{M} E(V-\mathcal{K})
$$

where $\mathcal{K}$ is the Kähler potential of $N$ and $V$ is the prepotential for the $U(1)$ gauge field introduced earlier. The equation of motion for $\phi$ is

$$
\nabla_{\alpha+} D_{+}^{\alpha} \phi^{r}=0 ; \quad r=1, \ldots \operatorname{dim}_{\mathrm{c}} \mathrm{N}
$$

where $\nabla$ includes the (pull-back of) the Levi-Civita connection on $N$. The equations of motion resulting from the variation of the supergravity fields can be expressed in the following simple form:

$$
F=\phi^{*} \Omega
$$

where $F$ is the $U(1)$ field strength two-form and $\Omega$ the Kähler two-form for $N$. These equations are completely equivalent to the component results obtained in section 4 as one may easily verify. One observes again the requirement that the target manifold should be Hodge as a consequence of the equations of motion. 


\section{7. (p,q) Poincaré theories}

The CS formulation of the general $(\mathrm{p}, \mathrm{q})$ Poincaré supergravity theory is found as follows. We first divide the $\mathrm{N}$ spinor supercharges of the MS supergravity theory into a set of $p$ charges $Q^{i}, i=1, \ldots, p$, and the complementary set of $q$ charges $Q^{i^{\prime}}, i^{\prime}=1, \ldots, q$, and introduce $p(p-1) / 2+q(q-1) / 2$ central charges, $Z^{i j}=-Z^{j i}$

and $Z^{i^{\prime} j^{\prime}}=-Z^{j^{\prime} i^{\prime}}$. We then define the (p,q)-Poincaré superalgebra to be the one for which the non-trivial (anti)commutators are

$$
\begin{aligned}
\left\{Q_{\alpha}^{i}, Q_{\beta}^{j}\right\} & =-\frac{1}{2} \delta^{i j}\left(\gamma^{a}\right)_{\alpha \beta} P_{a}+i \epsilon_{\alpha \beta} Z^{i j} \\
\left\{Q_{\alpha}^{i^{\prime}}, Q_{\beta}^{j^{\prime}}\right\} & =-\frac{1}{2} \delta^{i^{j^{\prime}}}\left(\gamma^{a}\right)_{\alpha \beta} P_{a}-i \epsilon_{\alpha \beta} Z^{i^{\prime} j^{\prime}} \\
{\left[M_{a}, M_{b}\right] } & =-\epsilon_{a b}{ }^{c} M_{c} \\
{\left[M_{a}, P_{b}\right] } & =-\epsilon_{a b}{ }^{c} P_{c} \\
{\left[M_{a}, Q_{\alpha}^{i}\right] } & =\frac{i}{2}\left(Q^{i} \gamma_{a}\right)_{\alpha} \\
{\left[M_{a}, Q_{\alpha}^{i^{\prime}}\right] } & =\frac{i}{2}\left(Q^{i^{\prime}} \gamma_{a}\right)_{\alpha} .
\end{aligned}
$$

When $p$ or $q$ is greater than unity, the $(\mathrm{p}, \mathrm{q})$ Poincaré superalgebra is a central extension of the N-extended Poincaré superalgebra. In this case is not possible to formulate a CS action for the algebra (7.1) because it does not admit a nondegenerate invariant inner product. To see this we note first that the inner product $\langle$,$\rangle of an even generator with an odd generator must vanish. Then, since$ $\langle$ even, odd $\rangle$ is both zero and invariant we deduce, in particular, that

$$
-\frac{1}{2} \delta^{i j}\left(\gamma^{a}\right)_{\alpha \beta}\left\langle P_{a}, Z^{k l}\right\rangle+\epsilon_{\alpha \beta}\left\langle Z^{i j}, Z^{k l}\right\rangle=0
$$

which implies that both $\langle P, Z\rangle$ and $\langle Z, Z\rangle$ vanish. Furthermore, the invariance of $\langle M, Z\rangle$ implies immediately that $\langle M, Z\rangle=0$. Similar arguments apply to the $Z^{\prime}$ generators so the only remaining way to achieve a non-degenerate invariant inner product would be to require $p=q$ and to pair the $Z$ with the $Z^{\prime}$ generators. But starting from the invariance of $\left\langle Q^{\prime}, Z\right\rangle$, a similar argument to the above one 
shows that $\left\langle Z, Z^{\prime}\right\rangle=0$. Thus, $Z$ and $Z^{\prime}$ are orthogonal to all generators of the $(\mathrm{p}, \mathrm{q})$ Poincaré superalgebra, including themselves, so any invariant inner product is degenerate. For this reason, we consider an enlarged superalgebra obtained in the following way. We first observe that the $(p, q)$ Poincaré superalgebra has an $S O(p) \times S O(q)$ automorphism group ${ }^{\star}$. We now take the semi-direct extension of this $s o(p) \oplus s o(q)$ automorphism algebra by the (p,q)-Poincaré superalgebra. Thus, we now include the new generators, $T^{i j}=-T^{j i}$, and $T^{i^{\prime} j^{\prime}}=-T^{j^{\prime} i^{\prime}}$, with the new non-trivial commutators as follows:

$$
\begin{aligned}
{\left[T^{i j}, Q^{k}\right] } & =2 \delta^{k[j} Q^{i]} \\
{\left[T^{i^{\prime} j^{\prime}}, Q^{k^{\prime}}\right] } & =2 \delta^{k^{\prime}\left[j^{\prime}\right.} Q^{\left.\prime i^{\prime}\right]} \\
{\left[T^{i j}, Z^{k l}\right] } & =2\left[\delta^{k[j} Z^{i] l}-(k \leftrightarrow l)\right] \\
{\left[T^{i^{\prime} j^{\prime}}, Z^{k^{\prime} l^{\prime}}\right] } & =2\left[\delta^{k^{\prime}\left[j^{\prime}\right.} Z^{\left.i^{\prime}\right] l^{\prime}}-\left(k^{\prime} \leftrightarrow l^{\prime}\right)\right] \\
{\left[T^{i j}, T^{k l}\right] } & =2\left[\delta^{k[j} T^{i] l}-(k \leftrightarrow l)\right] \\
{\left[T^{i^{\prime} j^{\prime}}, T^{k^{\prime} l^{\prime}}\right] } & =2\left[\delta^{k^{\prime}\left[j^{\prime}\right.} T^{\left.i^{\prime}\right] l^{\prime}}-\left(k^{\prime} \leftrightarrow l^{\prime}\right)\right]
\end{aligned}
$$

Note that although the charges $Z$ and $Z^{\prime}$ are central in (7.1) they are no longer central in the extended algebra including (7.3). The new superalgebra admits an invariant non-degenerate inner product, for which the non-vanishing components are

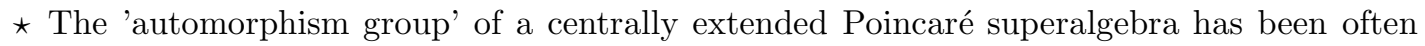
defined in the supersymmetry literature as that subgroup of the automorphism group of the algebra without central charges that commutes with the central charges. Here we adopt the standard mathematical terminology in which the central charges of an algebra need not be invariant under that algebra's automorphism group. 


$$
\begin{aligned}
\left\langle M_{a}, P_{b}\right\rangle & =\mu \eta_{a b} \\
\left\langle M_{a}, M_{b}\right\rangle & =\lambda \eta_{a b} \\
\left\langle Q_{\alpha}^{i}, Q_{\beta}^{j}\right\rangle & =i \mu \varepsilon_{\alpha \beta} \delta^{i j} \\
\left\langle Q_{\alpha}^{i^{\prime}}, Q_{\beta}^{j^{\prime}}\right\rangle & =i \mu \varepsilon_{\alpha \beta} \delta^{i^{\prime} j^{\prime}} \\
\left\langle Z^{i j}, T^{k l}\right\rangle & =-2 \mu \delta^{k[i} \delta^{j] l} \\
\left\langle Z^{i^{\prime} j^{\prime}}, T^{k^{\prime} l^{\prime}}\right\rangle & =2 \mu \delta^{k^{\prime}\left[i^{\prime}\right.} \delta^{\left.j^{\prime}\right] l^{\prime}} \\
\left\langle T^{i j}, T^{k l}\right\rangle & =2 \xi \delta^{k[i} \delta^{j] l} \\
\left\langle T^{i^{\prime} j^{\prime}}, T^{k^{\prime} l^{\prime}}\right\rangle & =2 \xi^{\prime} \delta^{k^{\prime}\left[i^{\prime}\right.} \delta^{\left.j^{\prime}\right] l^{\prime}}
\end{aligned}
$$

where $\mu$ is a non-zero real constant and, $\lambda, \xi$ and $\xi^{\prime}$ are arbitrary real constants. We introduce the gauge field one-forms associated with the generators of this enlarged superalgebra via the connection one-form

$a=\omega^{a} M_{a}+e^{a} P_{a}+\frac{1}{2} C_{i j} Z^{i j}+\frac{1}{2} C_{i^{\prime} j^{\prime}}^{\prime} Z^{\prime i^{\prime} j^{\prime}}+\frac{1}{2} A^{i j} T_{i j}+\frac{1}{2} A^{\prime i^{\prime} j^{\prime}} T^{\prime}{ }_{i^{\prime} j^{\prime}}+\psi^{i} Q_{i}+\psi^{i^{\prime}} Q_{i^{\prime}}$,

where the coefficients of the generators are the one-form gauge potentials. The corresponding field strength $F=d a+a^{2}$ is

$$
\begin{aligned}
F=T^{a} P_{a}+ & F^{a}(\omega) M_{a}+\frac{1}{2} F^{i j}(A) T_{i j}+\frac{1}{2} F^{i^{\prime} j^{\prime}}\left(A^{\prime}\right) T_{i^{\prime} j^{\prime}} \\
& +\frac{1}{2} G^{i j}(C) Z_{i j}+\frac{1}{2} G^{i^{\prime} j^{\prime}}\left(C^{\prime}\right) Z_{i^{\prime} j^{\prime}}+\left[\mathcal{D} \psi^{i}\right] Q^{i}+\left[\mathcal{D}^{\prime} \psi^{i^{\prime}}\right] Q^{i^{\prime}},
\end{aligned}
$$

where 


$$
\begin{aligned}
T^{a} & =d e^{a}-\epsilon_{b c}^{a} \omega^{b} e^{c}-\frac{1}{4} \bar{\psi}^{i} \gamma^{a} \psi^{i}-\frac{1}{4} \bar{\psi}^{i^{\prime}} \gamma^{a} \psi^{i^{\prime}} \\
F^{a}(\omega) & =d \omega^{a}-\frac{1}{2} \epsilon_{b c}^{a} \omega^{b} \omega^{c} \\
F^{i j}(A) & =d A^{i j}+A^{i k} A^{k j} \\
F^{i^{\prime} j^{\prime}}\left(A^{\prime}\right) & =d A^{i^{\prime} j^{\prime}}+A^{i^{\prime} k^{\prime}} A^{k^{\prime} j^{\prime}} \\
G^{i j}(C) & =d C^{i j}+C^{i k} A^{k j}+A^{i k} C^{k j}-i \bar{\psi}^{i} \psi^{j} \\
G^{i^{\prime} j^{\prime}}\left(C^{\prime}\right) & =d C^{i^{\prime} j^{\prime}}+C^{i^{\prime} k^{\prime}} A^{k^{\prime} j^{\prime}}+A^{i^{\prime} k^{\prime}} C^{k^{\prime} j^{\prime}}+i \bar{\psi}^{i^{\prime}} \psi^{j^{\prime}} \\
\mathcal{D} \psi^{i} & =d \psi^{i}+\frac{i}{2} \omega^{c} \gamma_{c} \psi^{i}+A^{i j} \psi^{j} \\
\mathcal{D}^{\prime} \psi^{i^{\prime}} & =d \psi^{i^{\prime}}+\frac{i}{2} \omega^{c} \gamma_{c} \psi^{i^{\prime}}+A^{i^{\prime} j^{\prime}} \psi^{j^{\prime}} .
\end{aligned}
$$

The CS action can be written, up to a surface term, as

$$
\begin{aligned}
S=\int d^{3} x[ & 2 \mu e_{a} F^{a}(\omega)+\lambda Q_{3}(\omega)-i \mu \bar{\psi}^{i} \mathcal{D} \psi^{i}-i \mu \bar{\psi}^{i^{\prime}} \mathcal{D} \psi^{i^{\prime}}-\frac{\xi}{2} Q_{3}(A)-\frac{\xi^{\prime}}{2} Q_{3}\left(A^{\prime}\right) \\
& \left.+\mu C^{j i} F^{i j}(A)-\mu C^{j^{\prime} i^{\prime}} F^{i^{\prime} j^{\prime}}\left(A^{\prime}\right)\right]
\end{aligned}
$$

where

$$
\begin{aligned}
Q_{3}(\omega) & =\omega_{a} d \omega^{a}-\frac{1}{3} \epsilon_{a b c} \omega^{a} \omega^{b} \omega^{c} \\
Q_{3}(A) & =A^{i j} d A^{j i}+\frac{2}{3} A^{i k} A^{k j} A^{j i} \\
Q_{3}\left(A^{\prime}\right) & =A^{i^{\prime} j^{\prime}} d A^{j^{\prime} i^{\prime}}+\frac{2}{3} A^{i^{\prime} k^{\prime}} A^{k^{\prime} j^{\prime}} A^{j^{\prime} i^{\prime}} .
\end{aligned}
$$

This action is invariant up to a surface term under the gauge transformation of the connection $a$. In particular, the non-zero supersymmetry transformation laws of the fields are

$$
\begin{aligned}
\delta e^{a} & =\frac{1}{2} \bar{\zeta}^{i} \gamma^{a} \psi^{i}+\frac{1}{2} \bar{\zeta}^{i^{\prime}} \gamma^{a} \psi^{i^{\prime}} \\
\delta C^{i j} & =-2 i \bar{\psi}^{[i} \zeta^{j]} \\
\delta C^{i^{\prime} j^{\prime}} & =2 i \bar{\psi}^{\left[i^{\prime}\right.} \zeta^{\left.j^{\prime}\right]} \\
\delta \psi^{i} & =\mathcal{D} \zeta^{i} \\
\delta \psi^{i^{\prime}} & =\mathcal{D}^{\prime} \zeta^{i^{\prime}}
\end{aligned}
$$


where $\zeta$ and $\zeta^{\prime}$ are the anticommuting spinor parameters. Observe that the automorphism gauge fields enter these transformations. It is this fact that accounts for the existence of Killing spinors for asymptotically conical spacetimes. This can be seen by repeating the analysis of section 3. One finds that Killing spinors exist provided that the holonomy of the $S O(p) \times S O(q)$ connections is reduced to a product of $U(1)$ factors. Note that the parameters $\lambda, \xi$ and $\xi^{\prime}$ appearing in the action (7.8) have dimension -1 in mass units, relative to $\mu$. It follows that the Lagrangian has a definite scaling weight only if $\lambda=\xi=\xi^{\prime}=0$. In this case (7.8) simplifies to

$$
S=\mu \int d^{3} x\left[2 e_{a} F^{a}(\omega)-i \bar{\psi}^{i} \mathcal{D} \psi^{i}-i \bar{\psi}^{i^{\prime}} \mathcal{D} \psi^{i^{\prime}}+C^{j i} F^{i j}(A)-C^{j^{\prime} i^{\prime}} F^{i^{\prime} j^{\prime}}\left(A^{\prime}\right)\right]
$$

which reduces to the action $(2.7)$ for the $(2,0)$ case. We remark that the above construction also works for 'non-standard' supersymmetry algebras with non-compact

automorphism groups, obtained by replacing the Kronecker deltas $\delta^{i j}, \delta^{i^{\prime} j^{\prime}}$ by the invariant tensors of non-compact versions of $S O(p) \times S O(q)$. In the $(3,0)$ case such a non-standard supergravity theory, with automorphism group $S L(2 ; \mathbb{R})$ instead of $S O(3)$, has the advantage that the holonomy matching condition for Killing spinors can be satisfied by cancelling the holonomy of the gravity sector against the holonomy of the gauge connection.

\section{8. adS supergravity and the Poincaré limit}

We now turn to the relation of the $(\mathrm{p}, \mathrm{q})$ Poincaré supergravity theories with the $(\mathrm{p}, \mathrm{q})$ adS theories. Unlike the Poincaré superalgebra, the adS superalgebras are semi-simple so the algebra of the outer automorphisms is isomorphic to the algebra of the inner automorphisms. It follows that the extended superalgebra that includes the automorphism generators is necessarily isomorphic to a direct sum of the adS superalgebra and its automorphism algebra. Consequently, nothing essential is gained in the formulation of the adS theories by the inclusion of automorphism generators. However, since the new (p,q) Poincaré supergravity theories include 
additional $S O(p) \times S O(q)$ gauge fields, it is clear that to obtain these Poincaré theories as limits of adS theories we must enlarge the adS superalgebras accordingly. We begin with the direct sum of the standard $(\mathrm{p}, \mathrm{q})$ adS superalgebra and an $s o(p) \oplus s o(q)$ algebra. The non-zero (anti)commutation relations are

$$
\begin{aligned}
{\left[M_{a}, M_{b}\right] } & =-\epsilon_{a b}{ }^{c} M_{c} \\
{\left[P_{a}, P_{b}\right] } & =-4 m^{2} \epsilon_{a b}{ }^{c} M_{c} \\
{\left[M_{a}, P_{b}\right] } & =-\epsilon_{a b}{ }^{c} P_{c} \\
\left\{Q_{\alpha}^{i}, Q_{\beta}^{j}\right\} & =-\frac{1}{2}\left(\gamma^{a}\right)_{\alpha \beta} P_{a} \delta^{i j}-m\left(\gamma^{a}\right)_{\alpha \beta} M_{a} \delta^{i j}+i m \epsilon_{\alpha \beta} Z^{i j} \\
\left\{Q_{\alpha}^{i^{\prime}}, Q_{\beta}^{j^{\prime}}\right\} & =-\frac{1}{2}\left(\gamma^{a}\right)_{\alpha \beta} P_{a} \delta^{i^{\prime} j^{\prime}}+m\left(\gamma^{a}\right)_{\alpha \beta} M_{a} \delta^{i^{\prime} j^{\prime}}-i m \epsilon_{\alpha \beta} Z^{i^{\prime} j^{\prime}} \\
{\left[M_{a}, Q_{\alpha}^{i}\right] } & =\frac{i}{2}\left(Q^{i} \gamma_{a}\right)_{\alpha} \\
{\left[P_{a}, Q_{\alpha}^{i}\right] } & =i m\left(Q^{i} \gamma_{a}\right)_{\alpha} \\
{\left[M_{a}, Q_{\alpha}^{i^{\prime}}\right] } & =\frac{i}{2}\left(Q^{i^{\prime}} \gamma_{a}\right)_{\alpha} \\
{\left[P_{a}, Q_{\alpha}^{i^{\prime}}\right] } & =-i m\left(Q^{i^{\prime}} \gamma_{a}\right)_{\alpha} \\
{\left[Z^{i j}, Q_{\alpha}^{k}\right] } & =2 \delta^{k[j} Q_{\alpha}^{i]} \\
{\left[Z^{i^{\prime} j^{\prime}}, Q_{\alpha}^{k^{\prime}}\right] } & =2 \delta^{k^{\prime}\left[j^{\prime}\right.} Q_{\alpha}^{\left.i^{\prime}\right]} \\
{\left[Z^{i j}, Z^{k l}\right] } & =2\left[\delta^{k[j} Z^{i] l}-(k \leftrightarrow l)\right] \\
{\left[Z^{i^{\prime} j^{\prime}}, Z^{k^{\prime} l^{\prime}}\right] } & =2\left[\delta^{k^{\prime}\left[j^{\prime}\right.} Z^{\left.i^{\prime}\right] l^{\prime}}-\left(k^{\prime} \leftrightarrow l^{\prime}\right)\right] \\
{\left[\bar{T}^{i j}, \bar{T}^{k l}\right] } & =-2 m\left[\delta^{k[j} \bar{T}^{i] l}-(k \leftrightarrow l)\right] \\
{\left[\bar{T}^{i^{\prime} j^{\prime}}, \bar{T}^{k^{\prime} l^{\prime}}\right] } & =-2 m\left[\delta^{k^{\prime}\left[j^{\prime}\right.} \bar{T}^{\left.i^{\prime}\right] l^{\prime}}-\left(k^{\prime} \leftrightarrow l^{\prime}\right)\right] .
\end{aligned}
$$

The mass parameter $m$ determines the scale of the cosmological constant. We now introduce the connection one-form

$\hat{a}=\omega^{a} M_{a}+e^{a} P_{a}+\frac{1}{2} A_{i j} Z^{i j}+\frac{1}{2} A_{i^{\prime} j^{\prime}}^{\prime} Z^{\prime i^{\prime} j^{\prime}}+\frac{1}{2} \bar{C}^{i j} \bar{T}_{i j}+\frac{1}{2} \bar{C}^{\prime i^{\prime} j^{\prime}} \bar{T}_{i^{\prime} j^{\prime}}^{\prime}+\psi^{i} Q_{i}+\psi^{i^{\prime}} Q_{i^{\prime}}$. 
The curvature two-form $\hat{F}=d \hat{a}+\hat{a}^{2}$ is

$$
\begin{aligned}
\hat{F}= & T^{a} P_{a}+\hat{F}^{a}(\omega) M_{a}+\frac{1}{2} \hat{F}^{i j}(A) Z_{i j}+\frac{1}{2} \hat{F}^{i^{\prime} j^{\prime}}\left(A^{\prime}\right) Z_{i^{\prime} j^{\prime}} \\
& +\frac{1}{2} \bar{G}^{i j}(\bar{C}) \bar{T}_{i j}+\frac{1}{2} \bar{G}^{i^{\prime} j^{\prime}}\left(\bar{C}^{\prime}\right) \bar{T}_{i^{\prime} j^{\prime}}+\Psi^{i} Q^{i}+\Psi^{i^{\prime}} Q^{i^{\prime}},
\end{aligned}
$$

where

$$
\begin{aligned}
\hat{F}^{a}(\omega) & =F^{a}(\omega)-2 m^{2} \epsilon_{a b c} e^{b} e^{c}-\frac{m}{2} \bar{\psi}^{i} \gamma_{a} \psi^{i}+\frac{m}{2} \bar{\psi}^{i^{\prime}} \gamma_{a} \psi^{i^{\prime}} \\
\hat{F}^{i j} & =F^{i j}(A)-i m \bar{\psi}^{i} \psi^{j} \\
\hat{F}^{i^{\prime} j^{\prime}} & =F^{i^{\prime} j^{\prime}}\left(A^{\prime}\right)+i m \bar{\psi}^{i^{\prime}} \psi^{i^{\prime}} \\
\Psi^{i} & =\mathcal{D} \psi^{i}+i m e^{a} \gamma_{a} \psi^{i} \\
\Psi^{i^{\prime}} & =\mathcal{D}^{\prime} \psi^{i^{\prime}}-i m e^{a} \gamma_{a} \psi^{i^{\prime}} \\
\bar{G}^{i j} & =d \bar{C}^{i j}-m \bar{C}^{i k} \bar{C}^{k j} \\
\bar{G}^{i^{\prime} j^{\prime}} & =d \bar{C}^{i^{\prime} j^{\prime}}-m \bar{C}^{i^{\prime} k^{\prime}} \bar{C}^{k^{\prime} j^{\prime}}
\end{aligned}
$$

and $T^{a}, F(\omega), F(A), F\left(A^{\prime}\right), \mathcal{D}$ and $\mathcal{D}^{\prime}$ are as defined in the previous section The algebra (8.1) has a class of invariant non-degenerate inner products depending on at least three and at most four parameters. This inner product is

$$
\begin{aligned}
\left\langle M_{a}, M_{b}\right\rangle & =\lambda \eta_{a b}, \quad\left\langle P_{a}, M_{b}\right\rangle=\mu \eta_{a b}, \quad\left\langle P_{a}, P_{b}\right\rangle=4 m^{2} \lambda \eta_{a b}, \\
\left\langle Q_{\alpha}^{i}, Q_{\beta}^{j}\right\rangle & =i(\mu+2 \lambda m) \epsilon_{\alpha \beta} \delta^{i j},\left\langle Q_{\alpha}^{i^{\prime}}, Q_{\beta}^{j^{\prime}}\right\rangle=i(\mu-2 \lambda m) \epsilon_{\alpha \beta} \delta^{i^{\prime} j^{\prime}}, \\
\left\langle Z^{i j}, Z^{k l}\right\rangle & =2\left(2 \lambda+\frac{\mu}{m}\right)\left(\delta^{i[l} \delta^{k] j}\right), \quad\left\langle Z^{i^{\prime} j^{\prime}}, Z^{k^{\prime} l^{\prime}}\right\rangle=2\left(2 \lambda-\frac{\mu}{m}\right)\left(\delta^{i^{\prime}\left[l^{\prime}\right.} \delta^{\left.k^{\prime}\right] j^{\prime}}\right), \\
\left\langle\bar{T}^{i j}, \bar{T}^{k l}\right\rangle & =2 \rho\left(\delta^{i[l} \delta^{k] j}\right), \quad\left\langle\bar{T}^{i^{\prime} j^{\prime}}, \bar{T}^{k^{\prime} l^{\prime}}\right\rangle=2 \rho^{\prime}\left(\delta^{i^{\prime}\left[l^{\prime}\right.} \delta^{\left.k^{\prime}\right] j^{\prime}}\right),
\end{aligned}
$$

where $\mu, \lambda$, and (for $p>1$ ) $\rho$ and (for $q>1$ ) $\rho^{\prime}$ are free real parameters. If one sets $m=0$ in (8.1) the resulting algebra is the semi-direct extension of $s o(p) \oplus s o(q)$ by the direct sum of the N-extended Poincaré superalgebra with an abelian algebra of dimension $\left[\frac{p(p-1)}{2}+\frac{q(q-1)}{2}\right]$. This contracted algebra is not isomorphic to the one given in section 2. However, it is known that the Wigner contractions of isomorphic algebras are not necessarily isomorphic [27] ; indeed, the algebra of 
section 5, defined by (7.1) and (7.3), results from a different contraction of (8.1). To see this, we use the redefinition

$$
T^{i j}=Z^{i j}-\frac{1}{m} \bar{T}^{i j}, \quad T^{i^{\prime} j^{\prime}}=Z^{i^{\prime} j^{\prime}}-\frac{1}{m} \bar{T}^{i^{\prime} j^{\prime}},
$$

to eliminate $Z$ and $Z^{\prime}$ in favour of $T$ and $T^{\prime}$. The non-trivial commutators involving the $T$ and $T^{\prime}$ generators are then

$$
\begin{aligned}
\left\{Q_{\alpha}^{i}, Q_{\beta}^{j}\right\} & =-\frac{1}{2}\left(\gamma^{a}\right)_{\alpha \beta} P_{a} \delta^{i j}-m\left(\gamma^{a}\right)_{\alpha \beta} M_{a} \delta^{i j}+i \epsilon_{\alpha \beta}(\bar{T}+m T)^{i j} \\
\left\{Q_{\alpha}^{i^{\prime}}, Q_{\beta}^{j^{\prime}}\right\} & =-\frac{1}{2}\left(\gamma^{a}\right)_{\alpha \beta} P_{a} \delta^{i^{\prime} j^{\prime}}+m\left(\gamma^{a}\right)_{\alpha \beta} M_{a} \delta^{i^{\prime} j^{\prime}}-i \epsilon_{\alpha \beta}(\bar{T}+m T)^{i^{\prime} j^{\prime}} \\
{\left[T^{i j}, Q_{\alpha}^{k}\right] } & =2 \delta^{k[j} Q_{\alpha}^{i]} \\
{\left[T^{i^{\prime} j^{\prime}}, Q_{\alpha}^{k^{\prime}}\right] } & =2 \delta^{k^{\prime}\left[j^{\prime}\right.} Q_{\alpha}^{\left.i^{\prime}\right]} \\
{\left[T^{i j}, T^{k l}\right] } & =2\left[\delta^{k[j} T^{i] l}-(k \leftrightarrow l)\right] \\
{\left[T^{i^{\prime} j^{\prime}}, T^{k^{\prime} l^{\prime}}\right] } & =2\left[\delta^{k^{\prime}\left[j^{\prime}\right.} T^{\left.i^{\prime}\right] l^{\prime}}-\left(k^{\prime} \leftrightarrow l^{\prime}\right)\right] \\
{\left[T^{i j}, \bar{T}^{k l}\right] } & =2\left[\delta^{k[j} \bar{T}^{i] l}-(k \leftrightarrow l)\right] \\
{\left[T^{i^{\prime} j^{\prime}}, \bar{T}^{k^{\prime} l^{\prime}}\right] } & =2\left[\delta^{k^{\prime}\left[j^{\prime}\right.} \bar{T}^{\left.i^{\prime}\right] l^{\prime}}-\left(k^{\prime} \leftrightarrow l^{\prime}\right)\right] .
\end{aligned}
$$

Similarly the components of the inner product involving $T$ and $T^{\prime}$ are

$$
\begin{aligned}
\left\langle T^{i j}, T^{k l}\right\rangle & =2\left(\frac{\rho}{m^{2}}+2 \lambda+\frac{\mu}{m}\right)\left(\delta^{i[l} \delta^{k] j}\right) \\
\left\langle T^{i^{\prime} j^{\prime}}, T^{k^{\prime} l^{\prime}}\right\rangle & =2\left(\frac{\rho^{\prime}}{m^{2}}+2 \lambda-\frac{\mu}{m}\right)\left(\delta^{i^{\prime}\left[l^{\prime}\right.} \delta^{\left.k^{\prime}\right] j^{\prime}}\right) \\
\left\langle T^{i j}, \bar{T}^{k l}\right\rangle & =-\frac{2 \rho}{m}\left(\delta^{i[l} \delta^{k] j}\right) \\
\left\langle T^{i^{\prime} j^{\prime}}, \bar{T}^{k^{\prime} l^{\prime}}\right\rangle & =-\frac{2 \rho^{\prime}}{m}\left(\delta^{i^{\prime}\left[l^{\prime}\right.} \delta^{\left.k^{\prime}\right] j^{\prime}}\right) .
\end{aligned}
$$

The $m \rightarrow 0$ limit of this algebra is the one of section 5. Moreover, the inner product used in section 5 for $\xi=-2 \lambda$ and $\xi^{\prime}=-2 \lambda$ can be obtained from the inner product defined above by setting $\rho=-m \mu$ and $\rho^{\prime}=m \mu$, and then taking 
the $m \rightarrow 0$ limit. The CS action of (p,q) adS supergravity based on the algebra (8.1) and the inner product (8.5) is, up to surface terms,

$$
\begin{aligned}
S= & \int d^{3} x\left[2 \mu e_{a} F^{a}(\omega)+\lambda Q_{3}(\omega)-\frac{4 m^{2} \mu}{3} \epsilon_{a b c} e^{a} e^{b} e^{c}+4 m^{2} \lambda e_{a} D e^{a}\right. \\
& -i(\mu+2 \lambda m)\left[\bar{\psi}^{i} \mathcal{D} \psi^{i}+i m \bar{\psi}^{i} \gamma_{a} e^{a} \psi^{i}\right]-i(\mu-2 \lambda m)\left[\bar{\psi}^{i^{\prime}} \mathcal{D}^{\prime} \psi^{i^{\prime}}-i m \bar{\psi}^{i^{\prime}} \gamma_{a} e^{a} \psi^{i^{\prime}}\right] \\
& \left.+\frac{1}{2}\left(2 \lambda+\frac{\mu}{m}\right) Q_{3}(A)+\frac{1}{2}\left(2 \lambda-\frac{\mu}{m}\right) Q_{3}\left(A^{\prime}\right)+\frac{\rho}{2} Q_{3}(\bar{C})+\frac{\rho^{\prime}}{2} Q_{3}\left(\bar{C}^{\prime}\right)\right]
\end{aligned}
$$

where

$$
\begin{aligned}
Q_{3}(\bar{C}) & =\bar{C} d \bar{C}-\frac{2}{3} m \bar{C}^{3} \\
Q_{3}\left(\bar{C}^{\prime}\right) & =\bar{C}^{\prime} d \bar{C}^{\prime}-\frac{2}{3} m \bar{C}^{\prime 3} \\
D e^{a} & =d e^{a}-\epsilon^{a}{ }_{b c} \omega^{b} e^{c} .
\end{aligned}
$$

To recover (p,q) Poincaré supergravity in the $m \rightarrow 0$ limit we set

$$
\bar{C}=C-\frac{1}{m} A \quad \bar{C}^{\prime}=C^{\prime}-\frac{1}{m} A^{\prime}
$$

and choose

$$
\rho=-m \mu \quad \rho^{\prime}=m \mu .
$$

In the $m \rightarrow 0$ limit we recover the action (7.11) with the parameters $\xi$ and $\xi^{\prime}$ equal to $-2 \lambda$. 


\section{Comments}

We have constructed a new class of $2+1$ Poincaré supergravity theories with $(\mathrm{p}, \mathrm{q})$ supersymmetry by including additional gauge fields associated with central charge and automorphism generators. In contrast to previous $\mathrm{N}=(\mathrm{p}+\mathrm{q})$ extended Poincaré supergravity theories, the new ones arise naturally as limits of an $S O(p) \times$ $S O(q)$ trivial extension of the $(\mathrm{p}, \mathrm{q})$-supersymmetric adS supergravity theories. In addition, both the $(1,1)$ and the $(2,0)$ Poincaré supergravity theories have an offshell superfield formulation. The new $(2,0)$ theory is analogous to the new-minimal formulation of $3+1$ supergravity. We have constructed the general coupling of this theory to sigma-model matter. Like the coupling to $\mathrm{D}=4 \mathrm{~N}=1$ supergravity, the target space is required to be a Hodge manifold. From the superfield point of view, the existence of distinct $N$-extended pure Poincaré supergravity theories with the same value of $N$ can be seen to be a consequence of the different possible choices of conformal compensating superfield. In $2+1$ dimensions this choice can lead to inequivalent theories as a result of the Chern-Simons structure of pure $2+1$ supergravity theories. For this reason we do not expect a similar phenomenon for conformal supergravity theories. One feature of the new Poincaré theories, e.g. $(2,0)$, is that the conical spacetimes of charged point particles admit Killing spinors for special values of the masses and charges; in this sense these spacetimes are the $2+1$ analogues of the Papapetrou-Majumdar multi charged black hole solutions of $3+1$ Maxwell/Einstein theory. The subdivision of the $N$-extended supergravity theories into $(\mathrm{p}, \mathrm{q})$ ones is inevitable in the adS case because of the structure of the adS superalgebra. For the reasons just explained, it is also natural in the Poincaré case, although here one can envisage a more general subdivision into partitions

$\left(p_{1}, p_{2}, \ldots, p_{k}\right)$ with $N=\sum_{i=1}^{k} p_{i}$. Such models can indeed be constructed, and the vacuum spacetimes again admit Killing spinors under suitable conditions. Finally, it is of obvious interest to quantize the new supergravity theories. The additional gauge fields will provide an additional finite number of degrees of freedom equal to the dimension of the moduli space of flat connections. 


\section{Acknowledgements}

G.P. is supported by a University Research Fellowship from the Royal Society.

J.M.I. thanks the Commission of the European Community and CICYT (Spain) for financial support.

\section{REFERENCES}

1. P. van Nieuwenhuizen, Phys. Rev. D32 (1985) 872;

J. Horne and E. Witten, Phys. Rev. Lett. 62 (1989) 501.

2. A. Achúcarro and P.K. Townsend, Phys. Lett. 180B (1986) 89.

3. E. Witten, Nucl. Phys. B311 (1988) 46.

4. N. Marcus and J.H. Schwarz, Nucl. Phys. B228 (1983) 145.

5. A. Achúcarro and P.K. Townsend, Phys. Lett. B229 (1989) 383.

6. S.W. Macdowell, The transformation algebra of fields in supergravity in proceedings of the Eighth International Conference on General Relativity and Gravitation, Waterloo, Ontario, Canada, 1977.

7. M. Roček and P. van Nieuwenhuizen, Class. Quantum Grav. 3 (1986) 43.

8. H. Nishino and S.J. Gates Jr., Int. J. Mod. Phys. A8 (1993) 3371.

9. M. Sohnius and P.C. West, Phys. Lett. 105B (1981), 353.

10. P.S. Howe, K.S. Stelle and P.K. Townsend, Phys. Lett. 107B (1981) 82.

11. R. D'Auria, P. Fré, P.K. Townsend and P. van Nieuwenhuizen, Ann. Phys. (N.Y.) 155 (1984) 423.

12. E. Witten, Int. J. Mod. Phys. A10 (1995) 1247.

13. J.M. Izquierdo and P.K. Townsend, Class. Quantum Grav. 12 (1995) 895,.

14. G.W. Gibbons and C.M. Hull, Phys. Lett. 109B (1982) 190.

15. E. Witten, Commun. Math. Phys. 80 (1981) 381. 
16. S. Deser, R. Jackiw and G. 't Hooft, Ann. Phys. (N.Y.) 152 (1984) 220.

17. B. de Wit, H. Nicolai and A. Tollsten, Nucl. Phys. B392 (1993) 3.

18. K. Becker, M. Becker and A. Strominger, Three-dimensional supergravity and the cosmological constant, preprint hep-th/9502107.

19. J. Bagger and E. Witten, Phys. Lett. 115B (1982) 202.

20. A. Comtet and G.W. Gibbons, Nucl. Phys. B299 (1988) 719.

21. B.R. Greene, A. Shapere, C. Vafa and S.T. Yau, Nucl. Phys. B337 (1990) 1.

22. P.S. Howe and M.I. Leeming, in preparation.

23. V. Ogievetsky and E. Sokatchev, Sov. J. Nucl. Phys. 32 (1980) 589.

24. J. Wess and B. Zumino, Phys. Lett. 74B (1978) 51.

25. P.S. Howe and M.I. Leeming, Class. Quantum Grav. 11 (1994) 2843.

26. D. Khetcelius and B. Zupnik, Yad Phys. 47 (1988) 1147.

27. V. Aldaya and J.A. de Azcárraga, Int. J. Theor. Phys. 24 (1985) 141. 